NASA Contractor Report 3021

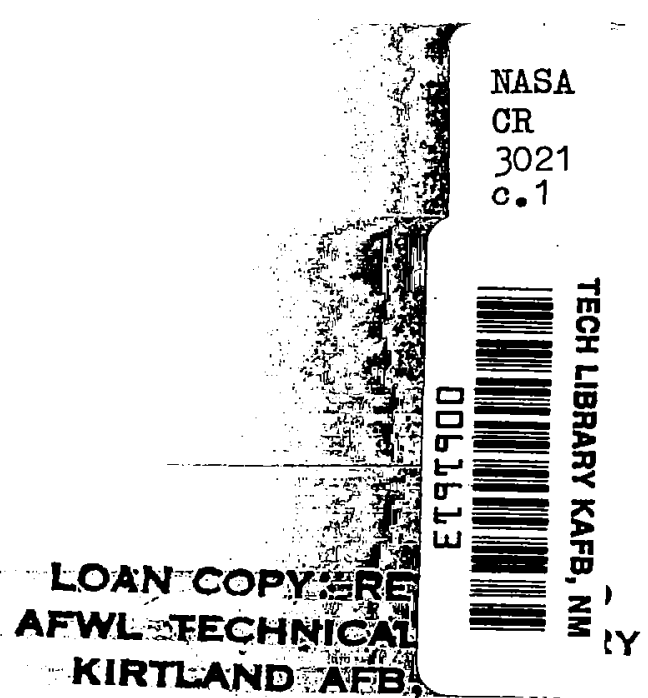

\title{
A Review of the Theory of Trailing Edge Noise
}

M. S. Howe

CONTRACT NAS1-14611

JUNE 1978 


\section{NASA Contractor Report 3021}

\section{A Review of the Theory of Trailing Edge Noise}

M. S. Howe

Bolt Beranek and Newman Inc. Cambridge, Massacbusetts

Prepared for Langley Research Center under Contract NAS1-14611

\section{N/Sก \\ National Aeronautics \\ and Space Administration}

\section{Scientific and Technical} Information Office 
TABLE OF CONTENTS

PAGE

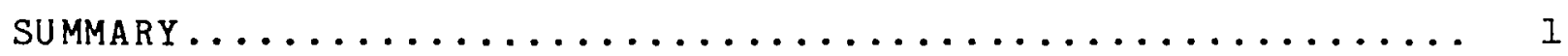

SECTION 1: INTRODUCTION....................... 2

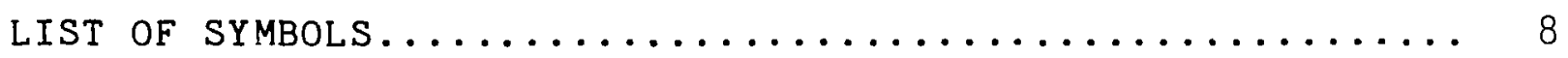

SECTION 2: EDGE NOISE THEORIES BASED ON LIGHTHILL'S

ACOUSTIC ANALOGY................... 12

SECTION 3: THEORIES BASED ON THE SOLUTION OF LINEARIZED

HYDROACOUSTIC EQUATIONS ................. 20

SECTION 4: AD HOC MODELS OF EDGE NOISE GENERATION........ 28

SECTION 5: A UNIFIED THEORY OF TRAILING EDGE NOISE....... 31

5.1: Solution of the Acoustic Analogy Equations..... 31

5.2: The Far Field Sound............... 38

5.3: The Principal Edge-Noise Dipole........... 40

5.4: Relation Between Surface Pressure

Fluctuations and the Far Field Sound......... 47

5.5: The Evanescent Wave Theory of Chandiramani

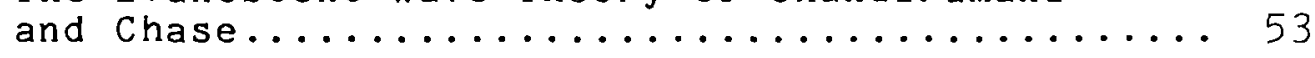

SECTION $6: \quad$ CONCLUSIONS ..................... 58

REFERENCES.......................... 60 


\section{LIST OF FIGURES}

PAGE

1(a). Configuration of the half-plane, the rectangular coordinate system $\left(x_{1}, x_{2}, x_{3}\right)$ and the observer

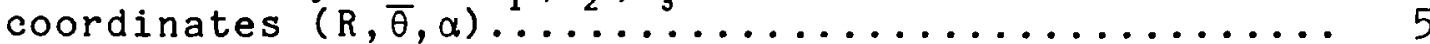

1(b). Turbulent boundary layer/wall jet flow past the trailing edge in a mean shear flow $\mathrm{V}\left(\mathrm{x}_{2}\right)$ which makes an angle $\beta$ with the positive $x_{1}$-axis, and wets a length $L$ of the edge. The mean flow far from the plate is at velocity $U_{0}$ in the positive $x$-direction and simulates forward flight at this

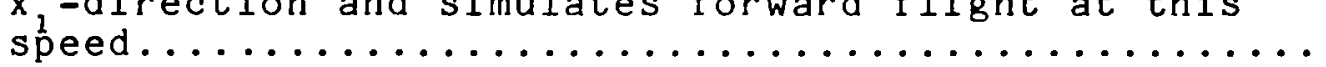

2(a). A line vortex of strength $\Gamma$ translates along the dashed-line path under the influence of a system of image vortices in the plate. The resulting unsteady motion is accompanied by the emission

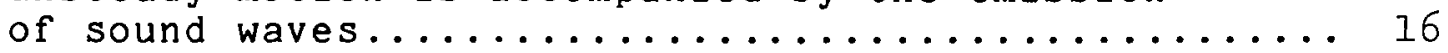

2(b). Application of the Kutta condition results in the shedding of voriticity $\gamma$ of opposite rotation to that of the incident vortex $\Gamma$. The intensity of radiated sound depends on the rate at which the vorticity translates across the parabolae $\Psi=$ constant. 17

3. Illustrating the mean flow configuration for linearized edge noise theories................

4. Comparison of measured edge noise spectra with that predicted by Chase (1975) for the

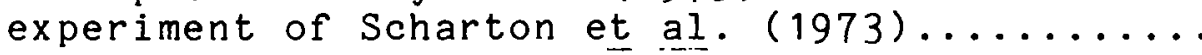

5. $O_{E}(r, \theta)$ is the location in the flyover-plane of the ground-fixed observer relative to the edge of the plate at the time of emission of the sound; $O_{A}(R, \bar{\theta})$ is the relative location at the time of arrival of the sound...

6. Comparison of the surface pressure spectrum near the traling edge (a) and the far field acoustic spectrum (b) at $\theta=90^{\circ}$ for the edge noise exper-

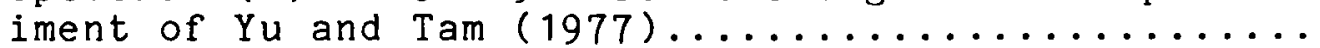


SUMMARY

This report presents a critical review of the literature on the theory of the generation of sound by the interaction of low Mach number turbulent flow with the edge of a semi-infinite rigid plate. Three distinct approaches to the subject are identified, consisting of theories based on (i) Lighthill's acoustic analogy; (ii) the solution of special, linearized hydroacoustic problems; (iii) ad hoc aerodynamic source models. It is shown that, when appropriately interpreted, all relevant theories produce essentially identical predictions in the limit of very small Mach numbers. None of the theories discusses the implications of the Kutta condition, however, nor of the effect of forward flight and source motion relative to the trailing edge. Accordingly the report includes an outline of a redevelopment of the theory which gives a unified view of the problem, exhibits the significance of the various approximations, and incorporates the effect of mean motion and of the Kutta condition. 
Sound is produced when unsteady flow interacts with a sharp-edged body such as a wing or flap. The noise generated by turbulence at the trailing edge of an airfoil can make a significant contribution to the overall sound radiated below the flight path of an aircraft (see, e.g., Fethney 1975; Hardin 1976). An important practical problem, therefore, concerns the development of procedures for predicting the characteristics of the radiated sound, such as its spectrum, field shape and the possible effects of forward motion of the aircraft. Ideally one would want to be able to express such predictions in terms of easily measured or estimated properties of the unsteady flow over the wing at flight Mach numbers corresponding to take-off and landing, and which are typically of order 0.3 or less. Thus flows of relatively low Mach numbers are of particular interest, although, as Crighton (1975) has emphasized, Doppler amplification can still exert a significant influence on the field shape of the radiated sound.

Data on the edge noise mechanism have been obtained from experiments in which the turbulent flow over the trailing edge is provided either by a wall jet (Hayden, Fox \& Chanaud 1976; Grosche 1970; Scharton, Pinkel \& Wilby 1973; Tam \& Reddy 1977; Yu \& Tam 1977), or by a low Mach number open wind tunnel flow containing grid turbulence (Fink 1975). These experiments were undertaken to determine the dependence of the acoustic field on fluctuating properties of the flow close to the trailing edge, and the scaling of the spectrum and overall sound pressure level (OASPL) with the mean flow velocity. The effect of flight on trailing edge noise has not been examined experimentally. In principle such an experiment could be performed in an open wind tunnel, for example, provided that the technical difficulties which have been encountered in analogous flight simulation tests on jet mixing noise can be overcome or shown to be unimportant (Fisher \& Morfey 1976).

The problem of trailing edge noise at low Mach numbers was first examined by Powell (1959). Similarity arguments were advanced to estimate the strength of postulated aerodynamic dipole sources located on the plate near the trailing edge. It was deduced from this mogel that the edge noise sound power varies approximatelyas!l-6, $U$ being the characteristic flow velocity, and that the power spectral density decays inversely as the cube of the frequency at high frequencies. Powell's treatment was not sufficiently detailed to predict the field shape of the radiation, nor could the effects of flight be considered.

The subsequent theoretical discussions of edge noise have been principally in terms of a prototype configuration which 
models the wing and trailing edge by a semi-infinite rigid plate, and consequently limits the validity of the conclusions to cases in which the nominal acoustic wavelength is small compared with the chord of the airfoil. The various published theories differ mainly in the way in which the fluctuating flow is assumed to interact with the edge of the plate to produce sound, and fall roughly into the following three categories:

(i) Theories based on the Lighthill (1952) acoustic analogy. These have been developed by Ffowcs Williams \& Hall (1970); Crighton \& Leppington (1970); Crighton (1972a); Levine (1975); Howe $(1975,1976,1977)$.

(ii) Theories based on the solution of special problems approximated by the linearized hydroacoustic equations: Crighton (1972b); Jones (1972); Crighton \& Leppington (1974); Morgan (1974); Chase (1972; 1975); Chandiramani (1974); Davis (1975); Amiet (1976).

(iii) Ad hoc models: these involve postulated source distributions whose strengths and multipole types are generally determined empirically: Hayden, Fox \& Chanaud (1976); Tam \& Yu (1975); Tam \& Reddy (1977).

In this report the edge noise theories listed above are discussed in detail. It is shown (Secs. 2-4) that when suitably viewed all relevant theoretical models lead to the same $U^{5}$ scaling law for the velocity dependence of the radiated sound, exhibit the same static directional characteristics and the same dependencies on the length scales of the turbulent edge flow. None of the theories gives an overall picture of the edge noise mechanism, however, in that those listed in (i) determine the radiation in terms of an assumed turbulent velocity field, while those in ( $i i)$ are confined to particular flow models or relate the sound field to pressure fluctuations on or near the edge of the plate.

The important question regarding the nature of the boundary condition at the trailing edge, viz., whether or not the unsteady flow should be required to leave the edge tangentially in accordance with the Kutta-Joukowski hypothesis, is considered in detail only by Jones (1972), Crighton (1972b), Davis (1975), and Howe $(1976,1977)$. Crighton's conclusion, that the imposition of the Kutta condition produces a dramatic increase in the level of the radiated sound, is now known to be incorrect (Crighton 1977, private communication); according to Howe's (1976, 1977) treatment of a series of two-dimensional model problems, the removal of the flow singularity at a sharp edge always leads to a reduction in the radiation, because the sound produced by the vorticity which must then be shed from the edge is of the 
appropriate phase and amplitude to cancel much of that generated by the incident turbulent flow. Yu and Tam (1977) have used a wall jet to investigate the nature of the flow at the trailing edge, and conclude that, at least at the relatively low Mach numbers of interest in the present discussion, vortices are shed into the wake in a manner which tends to oppose the production of a singular edge flow by the incident turbulence.

In Sec. 5, the detailed conclusions of the diverse theories reviewed in this report are condensed into a single theoretical model. This model exhibits:

I. the dependence of edge noise on the turbulent velocity field near the edge;

II. the relation between the acoustic spectrum and pressure fluctuations near the edge;

III. the significance of applying the Kutta condition;

IV. the effect of forward flight.

The principal conclusions are summarized in sec. 6 .

The geometry and coordinate system to be used in the discussion are depicted in Fig. 1. A rigid half-plane occupies the region $\left(x_{1}<0, x_{2}\right.$ a 0$)$ of a rectangular coordinate system $\left(x_{1}, x_{2}, x_{3}\right)$ and is located in fluid whose mean velocity far from the plate is $U_{0}$ parallel to the positive $x_{1}$ direction. Turbulence is convected past the edge of the plate in a boundary layer or wall jet flow whose mean velocity $V$ makes an angle $\beta$ with the positive $x_{1}$-axis, where $\underset{V}{V} V(\cos \beta, 0, \sin \beta) \equiv\left(V_{1}, 0, V_{3}\right)$, say.

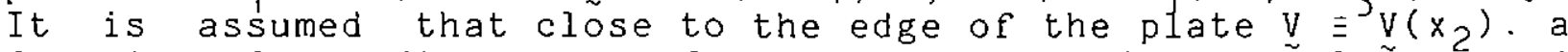
function of the distance $x_{2}$ from the surface of the plat̃e, and that the turbulent flow wets a length $L$ of the edge. Mach numbers $M_{O}, \underset{\sim}{M}$ are defined by

$$
\begin{aligned}
& \because_{0}=\frac{U_{0}}{c} \\
& M_{V} \equiv\left(M_{V_{1}}, 0, m_{V_{3}}\right) \equiv \frac{V}{c}
\end{aligned}
$$




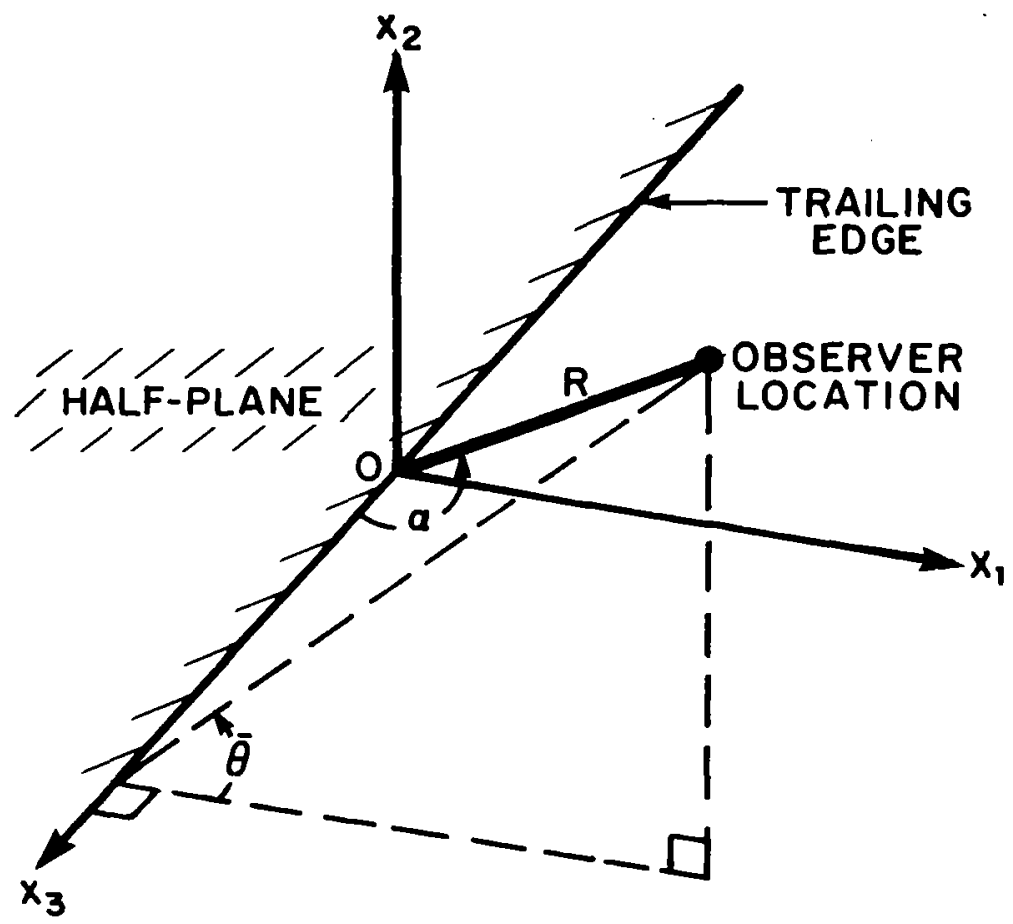

FIG. 1(a) CONFIGURATION OF THE HALF-PLANE, THE RECTANGULAR COORDINATE SYSTEM $\left(x_{1}, x_{2}, x_{3}\right)$ AND THE OBSERVER COORDINATES $(R, \bar{\theta}, \alpha)$. 


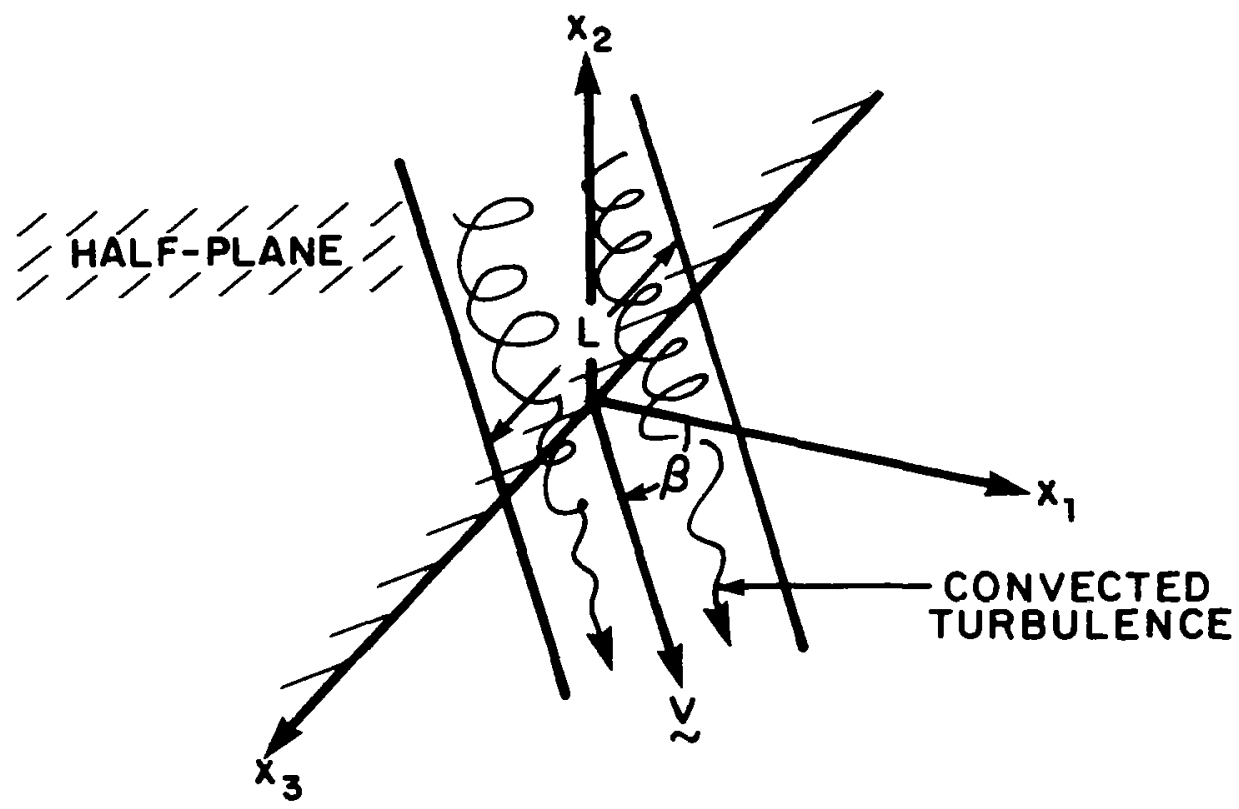

FIG. 1 (b) TURBULENT BOUNDARY LAYER/WALL JET FLOW PAST THE TRAILING EDGE IN A MEAN SHEAR FLOW $V\left(x_{2}\right)$ WHICH MAKES AN ANGLE $\beta$ WITH THE POSITIVE $\tilde{x}_{1}$-AXIS, AND WETS A LENGTH L OF THE EDGE. THE MEAN FLOW FAR FROM THE PLATE IS AT VELOCITY $U_{0}$ IN THE POSITIVE $x_{2}$-DIRECTION AND SIMULATES FORWARD FLIGHT AT THIS SPEED. 
where $c$ is the speed of sound, and are taken to be sufficiently small that $M_{0}{ }^{2}, M_{v}{ }^{2}$ may be neglected relative to unity, so that $c$ may be regarded as constant. In this respect note that mean flow effects arise in two ways: terms linear in $M$ account for the mean convection of the aerodynamic sound sources and of the emitted radiation; second, terms quadratic in $M$ describe the dynamic effect of compressibility on the mean flow. We ignore the latter, and systematically discard quantities of $O\left(M^{2}\right)$ relative to unity.

The author gratefully acknowledges the benefit of discussions with K.L. Chandiramani, D.M. Chase, D.G. Crighton, J.E. Ffowcs Williams, and R.E. Hayden during the preparation of this report. 


\section{Roman}

a

$B, B$

c

C, $\mathrm{C}_{v}, \overline{\mathrm{C}}_{v}, \mathrm{C}_{x}$

D

D

f

F

G

$\mathrm{k}$

L

$\ell$

$\ell_{0}$

$e_{j}(j=I, 2,3)$

$\mathrm{M}$

$\stackrel{M}{\sim W}$

$\stackrel{\mathrm{M}}{\sim}$

$\mathrm{M}_{0}$

$\stackrel{\mathrm{n}}{\sim}$

$\stackrel{N}{\sim}$

\section{LIST OF SYMBOLS}

distance of closest approach to edge of plate of the vortex of Figure 2(a)

stagnation enthalpy

speed of sound

numerical coefficients

nozzle exit diameter

dipole strength

pseudo-sound wavenumber spectrum

laminar sublayer transition function

Green's function

wavenumber variable

wetted length of trailing edge

characteristic spacing of eddies

length scale of the mean boundary layer/ wall jet profile

turbulence correlation scale in the $j$-direction

characteristic flow Mach number U/C

$W / \mathrm{c}$

$\mathrm{V} / \mathrm{c}$

$\mathrm{U}_{0} / \mathrm{c}$

unit vector parallel to $\mathrm{V}$

wavenumber vector defined in (5.17) 


\section{LIST OF SYMBOLS (Cont.)}

$p_{i}$

$\mathrm{p}_{\mathrm{K}}, \mathrm{p}_{\mathrm{I}}$

$\overline{\mathrm{p}}$

$P$

$q_{0}$

$\underset{\sim}{Q}, \underset{\sim}{q}$

$\hat{\mathrm{Q}}, \underset{\sim}{\hat{\mathrm{q}}}, \hat{\mathrm{p}}$

$\mathrm{S}, \mathrm{S}_{\mathrm{I}}, \mathrm{S}_{\mathrm{K}}, \mathrm{S}_{\mathrm{Kr}}, \mathrm{S}_{\mathrm{Ir}}$

$\mathrm{U}$

$\mathrm{U}^{\mathrm{J}}$

$\mathrm{U}_{0}$

$\underset{\sim}{\mathrm{V}}$

$\underset{\sim}{V} \equiv\left(V_{1}, 0, V_{3}\right)$

$\overline{\mathrm{V}}$

$v$

$\underset{\sim}{W} \equiv\left(W_{1}, 0, W_{3}\right)$

$x_{j}, X_{j}, Y_{j}$

$(j=1,2,3)$

Greek

$\beta$

$\gamma, \Gamma$ pseudo-sound pressure

pressure fluctuation with/without Kutta condition

root mean square hydrodynamic pressure fluctuation

overall acoustic frequency spectrum density

mean dynamic pressure

vorticity dipole strength

Fourier transform of $\underset{\sim}{Q}, \underset{\sim}{q}, p$

acoustic pressure frequency spectrum density

characteristic flow velocity

nozzle exit velocity

mean stream/flight speed

velocity

mean boundary layer/wall jet velocity

mean eddy convection velocity

root mean square turbulence velocity

near wake mean velocity

rectangular coordinates angle between $\underset{\sim}{\mathrm{V}}$ and $\mathrm{x}_{1}$-axis

vortex strength 


\section{LIST OF SYMBOLS (cont.)}

$$
\begin{aligned}
& \gamma(\mathrm{k}), \Gamma(\mathrm{k}) \\
& \delta, \delta_{\mathrm{n}}
\end{aligned}
$$

$\delta *$

$\Delta$

$\Delta_{E}$

$\lambda$

$\underset{\sim}{\mu}, \underset{\sim}{\mu}$

$v_{1}$

$\Pi, \Pi^{*}, \Pi_{i}, \Pi_{K}, \Pi_{I}$

$\rho, \rho_{0}$

$\tau$

$\sigma$

u

$\phi$

$\Phi_{i}, \Phi_{f}, \Phi_{3} 3$

$x$

$\Psi$

$\omega, \Omega$

$w_{z}$

$\underset{\sim}{\omega}, \underset{\sim}{\omega}$ $\mathrm{x}_{2}$-wavenumber component

turbulence correlation scale

laminar sublayer thickness

characteristic eddy volume

rms volume of edge noise source region

wavenumber magnitude in $(1,2)$-plane

wavenumber, vector

$\mathrm{x}_{1}$-component of shed vorticity wavenumber

surface pressure spectrum

density

retarded time

$\mathrm{W} / \mathrm{V}$

numerical constant

velocity potential

spectrum function

spectrum function

stream function

radian frequency

$\underset{\sim}{\mu} \cdot \underset{\sim}{\mathrm{V}}$

vorticity 


\title{
LIST OF SYMBOLS (Cont.)
}

\author{
Mixed \\ $(R, \bar{\theta}, \alpha)$ \\ $(r, \Theta, \Phi)$ \\ observer location at arrival time of sound \\ [see Figure I(a)] \\ observer location at time of emission of \\ sound
}




\section{SECTION 2 EDGE NOISE THEORIES BASED ON LIGHTHILL'S ACOUSTIC ANALOGY}

Ffowcs Williams and Hall (1970) have considered the infinite Reynolds number limit of Lighthill's (1952) equation in the case of isentropic turbulent flow in which the ambient medium is at rest; $U_{0}=0$, and perturbations in the pressure $p$ are related to density fluctuations $\rho-\rho_{0}$, by

$$
p=c^{2}\left(p-\rho_{0}\right)
$$

where $\rho_{0}$ denotes the mean density.

The starting equation for their analysis was accordingly

$$
\frac{1}{c^{2}} \frac{\partial^{2} p}{\partial t^{2}}-\nabla^{2} p=\frac{\partial^{2}}{\partial x_{1} \partial x_{j}}\left(\rho v_{i} v_{j}\right)
$$

where $v_{i}$ is the fluid velocity and the time. This equation was solved for an assumed knowledge of turbulent Reynolds stress $\rho v_{i} v_{j}$ by making use of a Green's function $G(\underset{\sim}{x}, \underset{\sim}{y}, t, \tau)$ which satisfies

$$
\frac{I}{c^{2}} \frac{\partial^{2} G}{\partial t^{2}}-\frac{\partial^{2} G}{\partial x_{j}^{2}}=\delta(t-\tau) \delta(\underset{\sim}{x}-\underset{\sim}{y}),
$$

the radiation condition of outgoing waves at infinity, and the rigid surface boundary condition

$$
\frac{\partial G}{\partial x_{2}}=0
$$

on the plate $\left(x_{1}<0, x_{2}=0\right)$. In the particular case of a plane, rigid boundary the condition $(2.4)$ is also satisfied by the pressure p.

By this means it was deduced that the far field mean square sound pressure $\left\langle\mathrm{p}^{2}\right\rangle$ produced by a compact turbulent eddy of 
volume $\Delta$ located well within a characteristic wavelength of the edge of the plate and translating at velocity $\mathrm{V}$ is given by

$$
\left\langle p^{2}\right\rangle \simeq \frac{\rho_{0}^{2} v^{2} V^{2} M}{\pi^{2}}\left(\frac{\delta}{R}\right)^{2}\left(\frac{\Delta}{\delta^{3}}\right)^{2} \sin \alpha \cdot \sin ^{2}\left(\frac{\bar{\theta}}{2}\right) \cdot \cos ^{2} \beta
$$

In this result

$$
\begin{aligned}
v^{2} & \text { mean square turbulent fluctuation velocity; } \\
\delta= & \text { characteristic turbulence correlation scale; } \\
R= & \text { distance of observer from the source region; } \\
\bar{\theta}= & \tan ^{-1}\left(\frac{\mathrm{x}_{2}}{\mathrm{x}_{1}}\right), \text { the "flyover" angle, }\left(\mathrm{x}_{1}, \mathrm{x}_{2}, \mathrm{x}_{3}\right) \text { being the } \\
& \text { observer coordinates (see Fig. } 1) ; \\
\alpha= & \text { angle between the observer direction and the edge of } \\
& \text { the half-plane. }
\end{aligned}
$$

According to (2.5) low Mach number turbulence near the edge of a rigid half-plane generates sound whose intensity exhibits the usual half-angle dependence on the "flyover" angle $\bar{\theta}$, peaking in the forward arc $\bar{\theta}>\pi / 2$, and scales as $U^{5}$, $U$ being a characteristic flow velocity; this may be coptrasted with the weaker dependencies of respectively $U^{6}$ and $U^{8}$ predicted for turbulent quadrupole sources located in the vicinity of a compact scattering body (Curle 1955) and in free space. In deriving (2.5) no attempt was made to estimate the influence of the back reaction of the plate on the turbulent flow. The authors argue that the prediction of a fifth-power law is a consequence of a potential field singularity which appears in their theory at the edge of the plate, and that the conclusion could be substantially modified if any type of Kutta condition were introduced to limit its effect. The infinite Reynolds number assumption must break down close to the trailing edge at sufficiently low frequencies, and it is difficult to see how the presence of a singularity can then be justified. Viscous forces may be invoked to remove the singularity, but as a consequence there will be unsteady vortex shedding from the edge resulting in the generation of additional turbulent fluctuations in the flow. In Sec. 5 the question of vortex shedding will be examined in detail, and it will be concluded that in general the radiation continues to scale as $U^{5}$ when the Kutta condition is imposed.

Expression (2.5) may easily be extended to take account of all of the turbulent eddies in the flow which contribute to the edge noise. Let $\delta_{n}$ denote the correlation scale of a typical eddy 
located near the edge of the half-plane. The lateral extent of all of theşe eddies is equal to $L$, and the effective mean square volume $\Delta_{E}$ of all eddies located within a correlation scale of the edge is therefore given by a summation over $L / \delta$ uncorrelated eddies. Thus we have

$$
\begin{aligned}
\Delta_{E}{ }^{2} & \simeq<\delta^{4}\left(\sum_{n} \delta_{n}\right)^{2}> \\
& \simeq \delta^{2}(L \delta)
\end{aligned}
$$

In addition, however, Ffowcs Williams \& Hall (1970) note (but do not exploit) the fact that the relative contributions from eddies arranged along a line parallel to the plate and perpendicular to the edge are weighted in inverse proportion to the square roots of their respective distances from the edge. The net result of this weighting is to introduce into the effective volume of the source region a factor proportional to $\sqrt{\cos \beta}$. Taking this and (2.6) into account we obtain the following order of magnitude edge noise prediction formula:

$$
\left\langle\mathrm{p}^{2}\right\rangle \simeq \rho_{0}^{2} v^{2} \mathrm{~V}^{2} \mathrm{M}_{\mathrm{V}}\left(\frac{\mathrm{L} \delta}{\mathrm{R}^{2}}\right) \sin \alpha \cdot \sin ^{2}\left(\frac{\bar{\theta}}{2}\right) \cos ^{3} \beta
$$

We shall argue below (Sec. 5) that this formula remains essentially unchanged when account is taken of vortex shedding, although it does not include $O(M)$ effects of aerodynamic source motion relative to the plate nor of aircraft flight.

Ffowcs Williams and Hall (1970) also obtained a $\mathrm{U}^{5}$ dependence for the case in which the disturbed flow satisfies a "pressure release" boundary condition on the plate, the latter being unable to support any normal stress. However, Crighton and Leppington (1970) have examined the problem for arbitrary surface compliance and their analysis shows that this prediction for the pressure release condition must be regarded as singular. They recovered the Ffowcs Williams and Hall result (2.7) only when the fluid loading of the plate was not significant and the plate relatively rigid. A quite different result obtains in cases of high fluid loading. The plate is now limp and the radiation is found to scale as $U^{6}$. Accordingly, the effect of surface compliance is to weaken near field scattering by the edge, but Crighton and Leppington show that in aeronautical applications only the first case considered by Ffowcs Williams and Hall (1970) of a rigid half-plane is likely to be relevant. 
In order to provide an explicit theoretical model of the edge noise mechanism Crighton (1972a) analyzed the two-dimensional flow illustrated in Fig. 2(a), involving an idealized turbulent eddy in the form of a line vortex of strength $\Gamma$ aligned parallel to the edge of a rigid half-plane. The mean flow is at rest relative to the plate and the vortex translates along the dashed-line path under the influence of a system of image vortices. The detailed motion of the vortex may be determined by assuming that the flow near the edge satisfies the equations of an ideal, incompressible fluid. The local unsteady potential flow thus defined may then be matched onto an acoustic disturbance which radiates away from the edge of the plate. In this way it was found that the intensity of the two-dimensional sound field scales in accordance with

$$
\left\langle\mathrm{p}^{2}\right\rangle \simeq \rho_{0}^{2} v^{4}\left(\frac{\mathrm{a}}{\mathrm{R}}\right) \sin ^{2}\left(\frac{\bar{\theta}}{2}\right)
$$

where $v=\Gamma / a$ is the characteristic fluctuation velocity, a denotes the distance of closest approach of the vortex to the edge of the half-plane, and ( $R, \bar{\theta})$ are polar coordinates of the observer in the $(1,2)-p l a n e$. Comparison may be made with the Ffowcs Williams-Hall result (2.7) if in the latter case the observer is assumed to lie in the "flyover"-plane $\alpha=\frac{1}{2}$, and the mean turbulent flow velocity $V$ is taken to be in the direction of the $x_{1}$-axis $(\beta=0)$. Both predictions are seen to agree apart from a factor of order $M(L / R)$ which accounts for the spherical spreading of sound waves from $L / \delta$ uncorrelated sources occupying a finite length $L$ parallel to the edge of the plate.

Howe (1975) re-worked the problem of Fig. 2(a) directly from the acoustic analogy theory of aerodynamic sound and deduced that at low Mach numbers the far field acoustic pressure could be expressed in the equivalent form

$$
p=\frac{\rho_{0} \Gamma \sin \frac{\bar{\theta}}{2}}{\pi \sqrt{R}}\left[\frac{D \psi}{\overline{D t}}\right],
$$

where

$$
\Psi=-\sqrt{\mathrm{R}_{0}} \cos \left(\frac{\bar{\theta}_{0}}{2}\right),
$$




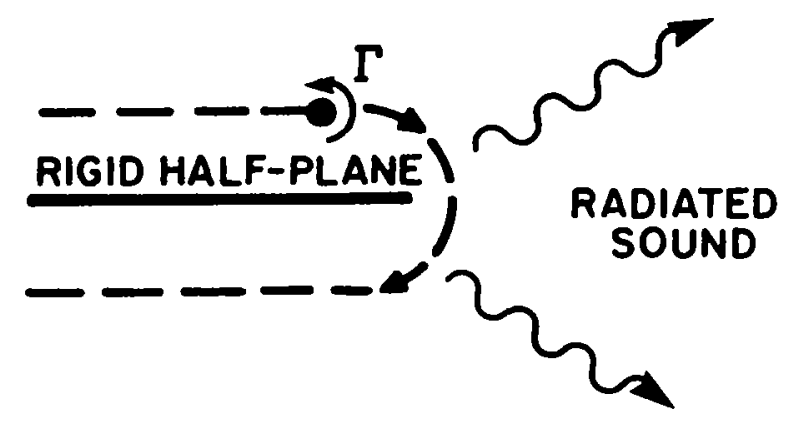

FIG. 2(a) A LINE VORTEX OF STRENGTH $\Gamma$ TRANSLATES ALONG THE DASHED-LINE PATH UNDER THE INFLUENCE OF A SYSTEM OF IMAGE VORTICES IN THE PLATE. THE RESULTING UNSTEADY MOTION IS ACCOMPANIED BY THE EMISSION OF SOUND WAVES. 


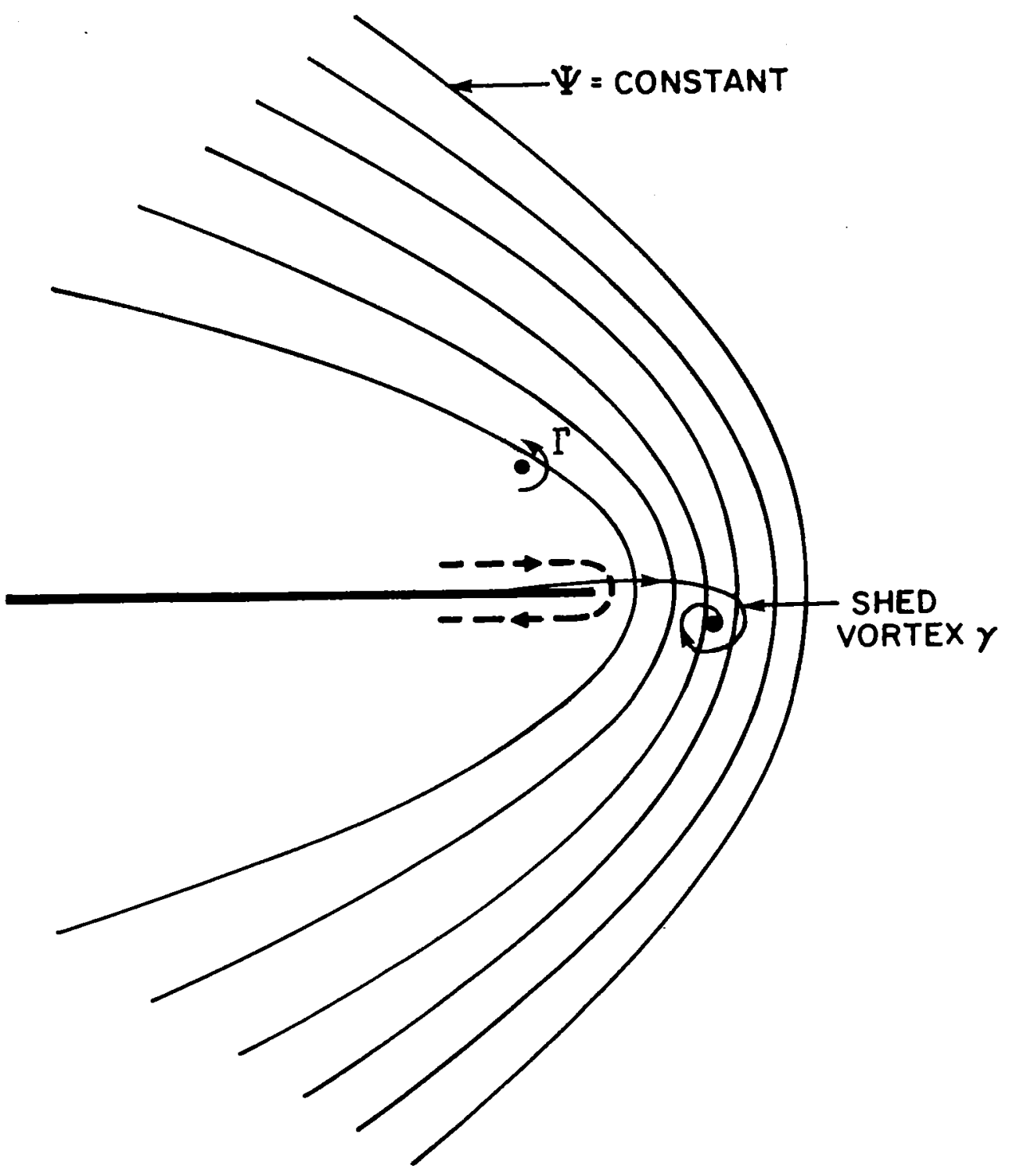

FIG. 2(b) APPLICATION OF THE KUTTA CONDITION RESULTS IN THE SHEDDING OF VORTICITY $\curlyvee$ OF OPPOSITE ROTATION TO THAT OF THE INCIDENT VORTEX $\Gamma$. THE INTENSITY OF THE RADIATED SOUND DEPENDS ON THE RATE AT WHICH THE VORTICITY TRANSLATES ACROSS THE PARABOLAE $\Psi=$ CONSTANT. 


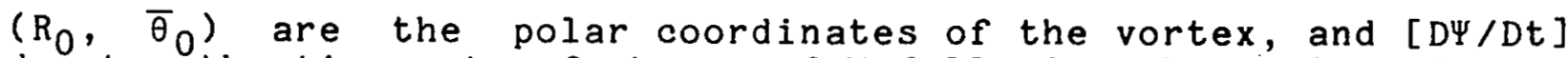
denotes the time rate of change of $\Psi$ following the motion of the vortex at the retarded time $t-R / c$. The function $\Psi$ assumes constant values along the family of parabolic curves illustrated in Fig. 2(b) which coincide with the streamlines of an ideal, source free two-dimensional potential flow about the half-plane. This type of flow can exist only in the absence of aerodynamic sources in the vicinity of the edge, i.e., when the edge is "silent". Eq. (2.9) reveals that the edge noise produced by the vortex is governed by the degree to which the actual flow near the edge departs from such a source-free potential flow, and this is measured by the rate at which the vortex translates across the family of "silent" streamlines.

This idealized description of the mechanism of edge noise generation in two-dimensional flows leads to a simple and elegant view of the effect of induced vortex shedding. Consider the situation illustrated in Fig. 2(b). The flow induced in an ideal fluid at the trailing edge by the vortex $\Gamma$ is in the sense indicated by the dashed curve, and possesses a singularity in velocity at the edge of the plate. The Reynolds number based on the boundary layer thickness at the edge is finite, and in practice the flow around the edge is impeded by the shedding of vorticity in a manner which tends to remove the velocity singularity. This requires the circulation $\gamma$ of the shed vorticity to have the opposite sense to that of the incident vortex $\Gamma$. Assume for simplicity that the principal component of the shed vorticity may be represented by a line vortex of strength $\gamma$. It then follows from (2.9) that the total radiated sound is given by

$$
p=\frac{\rho_{0} \sin \left(\frac{\bar{\theta}}{2}\right)}{\pi \sqrt{R}}\left\{\Gamma\left[\frac{D \Psi}{D t}\right]_{\Gamma}+\gamma\left[\frac{D \Psi}{D t}\right]_{\gamma}\right\}
$$

where the material derivatives are evaluated respectively at the positions of the incident and shed vortices. Both the incident and shed vorticity translate across the $\psi$-parabolae in essentially the same sense. Hence the material derivatives in (2.11) have the same sign, but $\Gamma$ and $\gamma$ are of opposite signs, from which we conclude that induced vortex shedding must reduce the overall level of the edge-noise. It will be shown in Sec. 5 that this is also expected to be the case in more general situations involving compact, three-dimensional turbulent eddies.

The problems illustrated in Fig. 2 have been generalized by Howe $(1976,1977)$ who applied the acoustic analogy theory to cases in which the incident vortex $\Gamma$ was constrained to move at constant velocity $V$ in the direction of the $x_{1}$-axis in the 
presence of a parallel, ambient mean flow of velocity $U_{0}\left(x_{2}\right)$. Several flow configurations were considered, including that in
which the mean velocity $U_{0}\left(x_{2}\right)$ assumed constant but different values on either side of the plate and of a vortex sheet extending downstream into the wake (c.f., Fig. 3). The mean fluid density $\rho$ was also permitted to be different on either side of the plate. In all of these cases it was found that when the Kutta condition was imposed at the trailing edge, so that the flow left the plate tangentially and additional vorticity was shed into the wake, the total edge scattered radiation was reduced to zero provided that the incident vortex translation velocity $V$ was just equal to that of the local mean flow in which it was located. When this happened the wake vorticity also convected at velocity $V$, and the sound it produced was equal and opposite to that generated by the incident vortex $\Gamma$. In addition it was shown that the degree of Doppler amplification of the radiation due to forward flight (and simulated in the analysis by the ambient mean flow velocity $U_{O}\left(x_{2}\right)$ ) depended critically on both the aerodynamic source velocity relative to the plate and on whether or not the Kutta condition was applied. Flight effects in relation to the edge noise problem have not been otherwise examined in the literature except for a recent paper by Crighton (1975) in which, however, no account was taken of source motion relative to the trailing edge.

The results of these various idealized problems suggest that induced vortex shedding tends to reduce the level of the radiated sound by increasing the effective length of the plate, i.e., of the boundary on which the normal velocity of the fluid vanishes. More specifically, in the absence of the Kutta condition there is an infinitely rapid transition from zero normal velocity to a singular normal velocity at the edge. Vortex shedding reduces the gradient of this transition, and, indeed, in the limiting case in which incident and shed vorticity convect downstream at the same constant speed, the normal velocity actually vanishes at all points of the plate and the wake (Howe, 1975, 1977), and no edge noise is produced.

Levine (1975) has examined a sequence of model problems in which the turbulent quadrupole strength $\rho v_{i} v_{j}$ was assumed to be constant in a frame convecting at a mean velocity $V$ in an arbitrary direction relative to the half-plane. It was shown that at low Mach numbers those quadrupoles with axes normal to the edge of the plate generate sound most efficiently (i.e., with sound pressure level scaling as $U^{5}$ ). The possibility of vortex shedding from the plate was not considered; Levine's results therefore corroborate the general conclusions which emerge from the Ffowcs Williams \& Hall (1970) theory. 
A variety of idealized, two-dimensional flow problems has been discussed by Crighton (1972b), Jones (1972), Crighton and Leppington (1974), Morgan (1974) and Davis (1975) in order to estimate the effect on edge noise of a wake extending downstream of the trailing edge and separating uniform mean flows at velocities $U_{1}, U_{2}$, say (Fig. 3). The analyses were based on the linearized equations of motion for which the wake was modeled by a linearly disturbed vortex sheet.

Crighton (1972b) considered the case in which $U_{1} \equiv 0$, and showed that, in the absence of aerodynamic sources outside of the wake, sound can be generated by the shedding of vorticity from the plate into the wake, the resulting fluctuating flow being singular at the trailing edge. The sound field may be calculated from Eq. (2.9) by supposing the wake to consist of a succession of line vortices aligned parallel to the edge of the plate and convecting in the downstream direction. For this case the sound pressure level scales according to the general two-dimensional result quoted above in (2.8). The vortex shedding actually triggers the Kelvin-Helmholtz instability of the vortex sheet whose amplitude of oscillation increases exponentially with distance downstream according to linear theory. This exponential growth may be shown to affect the phase but to have no essential influence on the amplitude of the radiation (Howe, 1976), and it is for this reason that linear theory may be expected to give a valuable first approximation to the edge noise interaction mechanism. Crighton has also examined the possibility of applying a Kutta condition to remove the edge singularity and, following earlier work of Orszag and Crow (1970), obtained a solution in which compressibility played a vital role in removing the singularity. The corresponding sound pressure level was found to scale as $U^{2}$, a much more significant velocity dependence than the previously predicted $\mathrm{U}^{4}$ result (2.8). The conclusion is erroneous, however, since an examination of Crighton's analysis reveals that the proposed solution does not satisfy the radiation condition of outgoing waves at infinity. The same error occurs in Davis's (1975) application of Crighton's method to the generation of high frequency edge noise by an airfoil set at zero incidence in a uniform stream (for which $U_{1} \equiv U_{2}$ ). Further, in considering various cases involving the excitation of a vortex sheet wake by a stationary line source, Jones (1972), crighton \& Leppington (1974) and Morgan (1974) discovered no significant effect on the radiation of imposing the Kutta condition. This is not in conflict with our earlier comments (Sec. 2) on the importance of the edge condition, which related to aerodynamic sources in motion relative to the plate. 


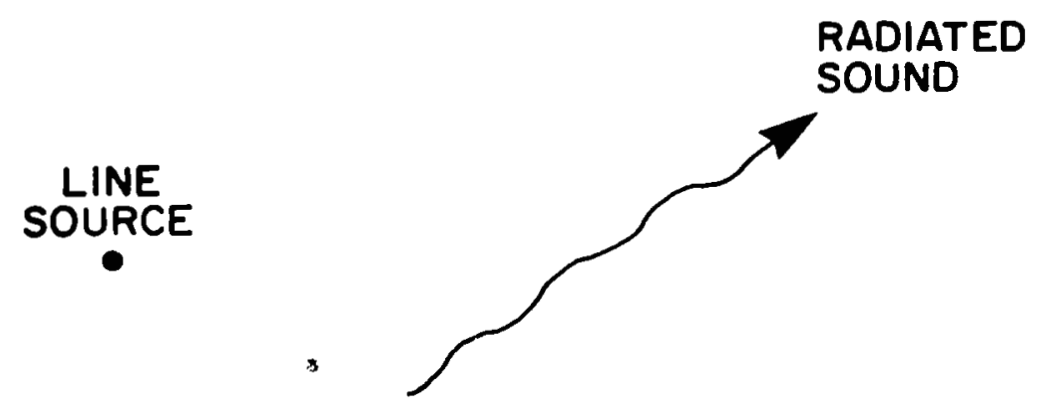

VORTEX SHEET WAKE

RIGID PLATE
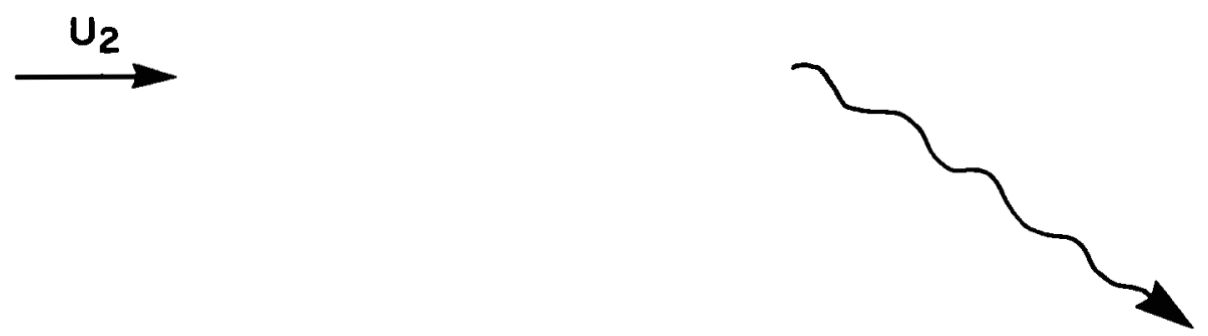

FIG. 3 ILLUSTRATING THE MEAN FLOW CONFIGURATION FOR LINEARIZED EDGE NOISE THEORIES. 
Chase $(1972,1975)$ and Chandiramani (1974) have proposed an alternative treatment of the edge noise problem with the object of relating the far field acoustic spectrum to measurable properties of the hydrodynamic pressure fluctuations on or near the trailing edge of the plate. Both authors implicitly consider only the case of a turbulent wall jet (Fig. 1) which does not wet the surface of the plate (i.e., such that the mean flow does not make contact with the surface), and take no account of forward flight nor of the possibility of vortex shedding.

Chase (1972) considers turbulent flow on the side $x_{2}>0$, say, of the plate. The half-plane Green's function which satisfies Eqs. $(2.3,2.4)$ above and which, in addition, vanishes on the downstream extension $S\left(x_{1}>0, x_{2}=0\right)$ of the plate is used, together with the assumption that the turbulent quadrupoles do not spill over the edge of the plate into $x_{2}<0$, to express the acoustic pressure $p(x, t)$ in $x_{2}<0$ in the form

$$
p(\underset{\sim}{x}, t)=\int_{S} p(\underset{\sim}{y}, \tau) \frac{\partial G}{\partial y_{2}}(\underset{\sim}{x}, \underset{\sim}{y}, t, \tau) d^{2} \underset{\sim}{y} d \tau,
$$

the integration being performed over all time $\tau$ and over the half-plane $S$. It is argued that linear diffraction theory predicts that the scattered pressure field vanishes identically on S (see, e.g., Noble 1958), and therefore the dominant contribution to $p(y, \tau)$ in (3.1) may be taken to be the linear component of the " pseudo-sound" pressure fluctuations produced by the turbulence in the absence of the plate. If it may be assumed that the turbulence is effectively frozen during its convection in the mean flow past the edge, the distribution $p(x, \tau)$ would then be equivalent to one half of the pseudo-sound pressure distribution induced on the plate when the same turbulence is located several hydrodynamic length scales upstream of the edge. That pressure field may accordingly be estimated from known characteristics of turbulent boundary layer flow over an extensive, flat rigid surface. Using (3.1) Chase (1972) is able to reproduce the Ffowcs Williams \& Hall result (2.7) to within a constant factor which is determined by the precise details of an assumed spectral distribution of the pseudo-sound field $p(y, \tau)$.

Chandiramani (1974) and Chase (1975) subsequently refined Chase's original argument by representing the pseudo-sound pressure fluctuations as a distribution of harmonic evanescent waves produced by convecting turbulent elements located above the plate in $x_{2}>0$, and given there by

$p_{i}(x, t)=\int \hat{p}_{i}\left(\mu_{1}, \mu_{3}, \omega\right) d \mu_{1} d \mu_{3} d \omega \cdot e$

$$
\left\{i\left(\mu_{1} x_{1}+\mu_{3} x_{3}-\omega t\right)+\left|\mu_{1}^{2}+\mu_{3}^{2}-\frac{\omega^{2}}{c^{2}}\right|^{1 / 2} x_{2}\right\} \text {. }
$$


The phase velocity of these waves parallel to the plate is subsonic and is taken to characterize the convection velocities of the turbulence in the wall jet. The Fourier coefficient $\hat{p}_{i}\left(\mu_{1}, \mu_{3}, \omega\right)$ is unknown but in principle may be estimated by first solving the diffraction problem of a rigid half-plane irradiated by the plane harmonic wave:

$$
\hat{p}_{1}\left(\mu_{1}, \mu_{3}, \omega\right) \exp \left\{1\left(\mu_{1} x_{1}+\mu_{3} x_{3}-\omega t\right)+\left|\mu_{1}^{2}+\mu_{3}^{2}-\frac{\omega^{2}}{c^{2}}\right|^{1 / 2} x_{2}\right\} .
$$

The solution determines both the far field edge scattered radiation produced by the evanescent wave and also the pressure distribution on the surface of the plate, and comparison of the latter with surface pressure measurements yields statistical information on $\hat{\mathrm{p}}_{i}\left(\mu_{1}, \mu_{3}, \omega\right)$. In practice it is more convenient to introduce an assumed form for the spectrum $\Pi_{i}\left(\mu_{1}, \mu_{3}, w\right)$ of the incident pseudo-sound, which satisfies

$$
\left\langle\hat{\mathrm{p}}_{i}\left(\mu_{1}, \mu_{3}, \omega\right) \hat{\mathrm{p}}_{i}\left(\bar{\mu}_{1}, \bar{\mu}_{3}, \Omega\right)\right\rangle=\Pi_{i}\left(\mu_{1}, \mu_{3}, \omega\right) \delta\left(\mu_{1}+\bar{\mu}_{1}\right) \delta\left(\mu_{3}+\bar{\mu}_{3}\right) \delta(\omega+\Omega)
$$

provided that the turbulent fluctuations are stationary random.

The frequency spectrum density $S(\omega)$, say, of the acoustic pressure satisfies

$$
\left\langle\mathrm{p}^{2}\right\rangle=\int_{0}^{\infty} S(\omega) \mathrm{d} \omega
$$

and in the particular case in which the distribution of the eddy convection velocity is centered about a mean value $v$, Chase (1975) deduced that

$$
S(\omega)=\frac{2 \mathrm{M}_{\mathrm{V}} \mathrm{L}}{\pi \mathrm{R}^{2}} \sin \alpha \sin ^{2}\left(\frac{\bar{\theta}}{2}\right) \cos \beta \int_{-\infty}^{\infty} \pi_{1}\left(\mu_{1}, \frac{\omega \cos \alpha}{c}, \omega\right) d_{\mu_{1}} .
$$

The integrand is sharply peaked at that value of $\mu_{j}$ for which $\omega=$ $\mathrm{V} \cdot \mathrm{\mu}$. In his analysis of the edge noise radiation from the wall jet studied experimentally by Scharton, Pinkel and Wilby (1973), 
Chase selected the following functional form for the pseudo-sound spectrum:

$$
\Pi_{i}\left(\mu_{1}, \mu_{3}, \omega\right)=\frac{v^{3} \rho_{0}^{2} C_{U} \delta^{5}(\underset{\sim}{\mu} \cdot n)^{2}}{\left\{1+\left(\mu_{1}^{2}+\mu_{3}^{2}\right) \delta^{2}+\left[\frac{(\omega-\underset{\sim}{V} \cdot \underset{\sim}{\mu}) \delta}{v}\right]^{2}\right\}^{U+1 / 2}}
$$

in which $U, C_{U}$ are dimensionless constants, $\underset{v}{n}$ is a unit vector parallel to a mean eddy convection velocity $V$ and the remaining symbols have been defined previously. Numerical values for the undetermined parameters $C_{U}, \underset{\sim}{V}, v, \delta$ and $U$ were obtained by comparison with surface pressure measurements. The acoustic power spectrum may be expressed in terms of $I I$ as in (3.5) and therefore in terms of the measured statistical properties of the surface pressure fluctuations. Only a relatively crude determination of $\Pi_{1}$ was possible from the experimental data, although the reported differences between measured and predicted 1/3-octave noise spectra were less than $5 \mathrm{~dB}$, the theory underestimating the actual spectral levels by at most this amount at frequencies less than the peak frequency (which occurred at a Strouhal number $\omega D / 2 \pi U \sim 0.3, D$ and $U$ being respectively the nozzle exit diameter and velocity of the jet: see Fig. 4). We shall argue below (Sec. 5) that an underestimate of this sort is not an entirely unexpected feature of the theory, and arises because of the unrealistic assumption that the jet does not wet the surface of the plate, from which the theory predicts that the fluctuating pressure remains finite at the trailing edge. In Sec. 5 , the effects of surface wetting will be incorporated into the theory, and it will be shown that finiteness of pressure can only be ensured when the full Kutta condition is imposed. We have already described how the application of this condition leads to an inevitable reduction in the predicted radiation levels. In practice it is likely that, in applying the idealized edge noise theories discussed in this report, an edge condition intermediate between the full Kutta condition and no Kutta condition should actually be imposed. Predictions based on applying the full Kutta condition would therefore be expected to yield lower bounds for the edge noise radiation.

For the general case specified by (3.6) the following representation of the mean square sound pressure in the far field can be deduced from Chase's (1975) analysis:

$\left\langle\mathrm{p}^{2}\right\rangle=\frac{\Gamma(u)}{\sqrt{\pi} \Gamma(u+I / 2)(2-u)(I-v)}\left(\frac{L \delta}{\mathrm{R}^{2}}\right) \mathrm{C}_{v} \rho_{0}^{2} v^{4} \mathrm{M}_{\mathrm{v}} \sin \alpha \sin ^{2}\left(\frac{\bar{\theta}}{2}\right) \cos ^{3} \beta, \quad(3.7)$ 


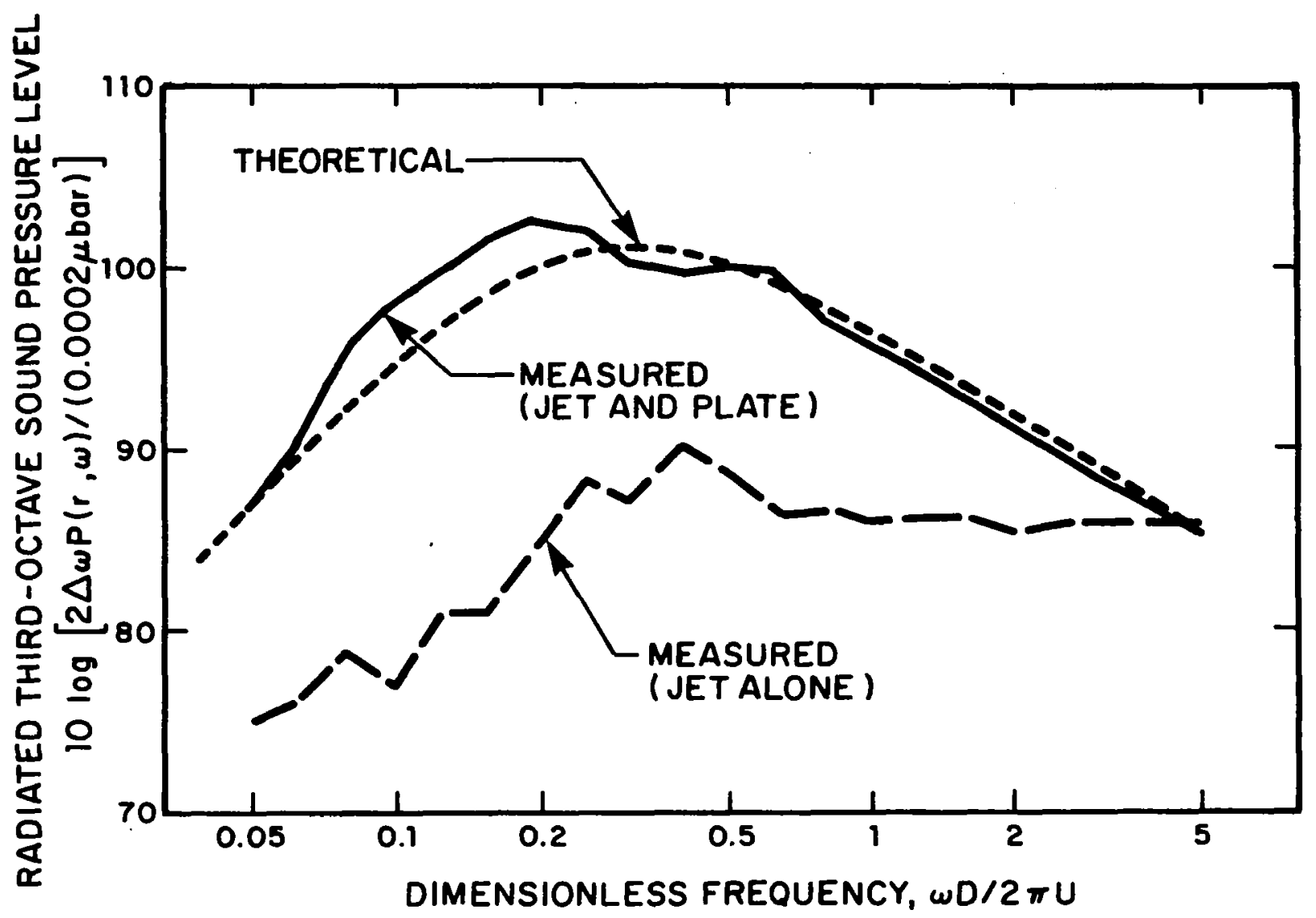

FIG. 4 COMPARISON OF MEASURED EDGE NOISE SPECTRA WITH THAT PREDICTED BY CHASE (1975) FOR THE EXPERIMENT OF SCHARTON ET AL. (1973). 
where $\Gamma(z)$ denotes Euler's $\Gamma$-function and $U$ must be assumed to exceed 2. This result is identical in form with the Ffowcs Williams \& Hall expression (2.7) derived directly from the acoustic analogy theory. Chase's corresponding formula for the power spectral density $S(\omega)$ is given by

$$
\begin{aligned}
S(\omega) & =\frac{2 \Gamma(U)}{\sqrt{\pi} \Gamma(U+I / 2)}\left(\frac{L \delta}{R^{2}}\right) C_{v} \rho_{0}^{2} v^{4} M_{v} \sin \alpha \sin ^{2}\left(\frac{\bar{\theta}}{2}\right) \\
& \times \frac{(\omega \delta / V)^{2}(\delta / V)}{\left[I+\left(\frac{\omega \delta \sec \beta}{V}\right)^{2}\right]^{U}},
\end{aligned}
$$

the mean square pressure $\left\langle\mathrm{p}^{2}\right\rangle$ of (3.7) being obtained by integration over all positive radian frequencies $\omega$.

Amiet (1976) has proposed an extension of Chase's theory to include the presence of an ambient mean flow $U_{0}$. The radiated field was determined by first estimating the strength of a distribution of surface dipoles on the plate from the calculated lift fluctuation produced by the incident disturbance (3.2). The dipole strength quoted by Amiet is in error, however, in particular it does not exhibit the expected surface pressure doubling far upstream of the edge, and detailed conclusions must therefore await a further development of Amiet's theory.

The generation of sound by turbulent boundary layer flow past a trailing edge was modeled by Chandiramani (1974), who chose the functional form of $\pi_{i}\left(\mu_{1}, \mu_{3}, \omega\right)$ on the basis of experimental data and the similarity hypothesis of Corcos (1964) for a turbulent boundary layer on an extensive, flat rigid surface. This type of boundary layer flow was assumed to characterize the incident pressure distribution $p_{i}(x, t)$ of $(3.2)$ and to exist on one side $\left(x_{2}>0\right.$, say) of the rigid pilate for $x_{1}$ $<0$ as well as for a short distance $(\sim 0(V / \omega))$ downstream of the edge. The mean flow was parallel to the $x_{1}$-direction and Chandiramani deduced that the spectrum of the total radiated sound power $P(\omega)$ defined by

$$
P(\omega)=\int_{\Sigma} \frac{S(\omega)}{\rho_{0} c} d \Sigma,
$$


the integration being over the surface $\Sigma$ of a large sphere of radius $R$ centered on the source region, can be represented by

$$
P(\omega) \simeq \mathrm{CM}_{\mathrm{v}} L \delta \Phi_{f}(\omega)
$$

Here $C$ is a dimensionless constant and $\Phi_{f}$ is the frequency spectrum of wall pressure fluctuations as measured by an ideal, point transducer several length scales upstream of the trailing edge.

The result (3.10) of Chandiramani and the corresponding formulae ( $3.5,3.8)$ of Chase are in general qualitative agreement. They are appropriate to the special case in which the radiated sound is generated principally by those eddies located in the vicinity of the "convective ridge" of the turbulence wavenumber-frequency spectrum. The applicability of the evanescent wave method is in no way restricted to this particular case, however, and remains valid for an arbitrary distribution of eddy convection velocity (c.f., Chase (1975) equation (5) and Sec. 5 below). 
Hayden, Fox and Chanaud (1976) have discussed a mechanism of edge noise generation which is a development of earlier work of Hayden (1969). Using arguments first proposed by Powell (1959), it is contended that the edge noise originates from a system of dipole sources located just downstream of the edge. The dipoles are orientated perpendicular to the plane of the plate and are as sumed to characterize the hydrodynamic interaction between the edge and incident turbulence including any additional effects associated with vortex shedding and/or shear layer instability.

Hayden et al. (1976) argue that the passage of a turbulent eddy past the trailing edge results in a mismatch in the forces which act upon the eddy, the stress exerted by the plate decaying rapidly to zero. Accordingly there is a tendency for the fluid to accelerate in a direction normal to the plate under the influence of a localized force whose strength per unit volume may be expressed in the form

$$
D=\frac{\rho_{0} V^{2}}{2 \delta}\left(\frac{\bar{p}}{q_{0}}\right),
$$

corresponding to an acceleration $D / \rho_{0}$. In this equation $\delta$ is the correlation scale of the eddy, $\bar{p}$ the characteristic root mean square fluctuating pressure and $\mathrm{q}_{0}$ is the mean dynamic pressure. Integration of (4.1) over the whole of the fluid yields the net force exerted on the fluid by the plate.

In order to calculate the radiated sound, an equivalent acoustic dipole is introduced whose strength is determined by comparing the mean square acceleration given by the order of magnitude formula (4.1) with that which such a dipole would induce in the flow at a radial distance $R \simeq \delta$ when located in free space. In their comparison, Hayden et al. erroneously retain contributions to the near field acceleration produced by the acoustic dipole which involve fluid compressibility, i.e., terms which depend on the ability of the dipole to launch acoustic waves. The latter is influenced dramatically by the presence of the half-plane, and a correct comparison of near fields which takes account of compressibility should properly include (i) the effect of compressibility on the postulated volume force (4.1), and (ii) the effect of the half-plane on the near field of the acoustic dipole. 
The correct interpretation of Hayden, Fox and. Chanaud's theory is that the gradient $\partial D / \partial x_{2}$ calculated from (4.1) actually constitutes the leading contribution to the Lighthill (1952) double divergence quadrupole source $\left.\partial^{2}\left(\rho v_{i} v_{j}\right) / \partial x_{i} \partial x_{j}\right)$ of Eq. (2.2). The radiated sound may then be computed in the manner prescribed by Ffowcs Williams \& Hall (1970) using the half-plane Green's function. Thus taking (4.1) to be the dipole strength of an eddy whose center is located in the wake at a distance d from the edge of the plate, we then obtain in the far field

$$
\left\langle\mathrm{p}^{2}\right\rangle \simeq \frac{\delta^{6}|D|^{2} \omega}{2 \pi \mathrm{R}^{2} \mathrm{dc}} \sin \alpha \cdot \sin ^{2}\left(\frac{\bar{\theta}}{2}\right)
$$

where $\omega$ is the characteristic source frequency.

In applying a result of this type to estimate the edge noise generated by a turbulent wall jet Hayden et al. (1976) set $\omega=$ $\mathrm{V} / \ell$ and $\mathrm{d}=\delta$, where $\ell$ is the characteristic distance between successive eddies of length scale $\delta$, and $V$ is the eddy convection velocity. But this procedure overlooks the fact that the contribution to the linear acoustic field furnished by a typical eddy decreases very slowly as a function of its distance d from the edge, the decay being proportional to $1 / \sqrt{d}$ (c.f., the discussion of the Ffowcs Williams and Hall theory following Eq. (2.6) above). When this is taken into account, by summing over all eddies along a line parallel to the plate and perpendicular to the edge, and when in addition a spanwise summation over the wetted length $L$ is performed, it is found that the overall mean square acoustic pressure is obtained from (4.2) by multiplying by a factor of order $\mathrm{VdL} / \omega \delta^{3}$, in which case we have

$$
\left\langle\mathrm{p}^{2}\right\rangle=\frac{\rho_{0}^{2} \mathrm{~V}^{4} \mathrm{M}}{8 \pi}\left(\frac{\mathrm{L} \delta}{\mathrm{R}^{2}}\right)\left(\frac{\overline{\mathrm{p}}}{\mathrm{q}_{0}}\right) \sin \alpha \cdot \sin ^{2}\left(\frac{\bar{\theta}}{2}\right),
$$

use having been made of (4.1). This corrected form of the Hayden, Fox and Chanaud prediction formula is in complete accord with the Ffowcs Williams and Hall result (2.7).

Tam and Reddy (1977) have discussed the possibility that the dominant source of trailing edge noise is not the mechanism discussed above, but rather the free turbulence generated in the wake as a result of flow past the trailing edge, the latter being unimportant as far as the production of sound is concerned. Numerical computations performed on this basis lead Tam and Redgy to the conclusion that the sound pressure level scales as $U^{6}$. 
This appears to be a mistaken prediction, arising from a failure to recognize the quadrupole nature of the turbulent wake sources.

Numerical calculations have been performed by Tam and Yu (1975) to determine the directivity of a dipole source located in the wake just downstream of the trailing edge of a plate of finite chord. The structure of the source was not discussed, the object being to illustrate the asymptotic manner in which the half-plane directivity was approached with increasing source frequency. Not surprisingly, it was found that as the wavelength of the sound progressively diminishes, the directivity fluctuates ever more rapidly with angle. In the flyover plane $\alpha=\pi / 2$, for example, the $\sin ^{2}(\bar{\theta} / 2)$ dependence of the Ffowcs Williams and Hall prediction should be interpreted as the envelope of the actual directivity. 
In this section we outline the development of a theory of trailing edge noise which incorporates as special cases the various approaches discussed above. In addition we shall examine how the application of the Kutta condition affects the predicted radiation levels and assess the Doppler amplification produced by source motion relative to the edge and by forward flight.

\section{SECTION 5.1 SOLUTION OF THE ACOUSTIC ANALOGY EQUATIONS}

The boundary layer/wall jet flow is assumed to be isentropic, at constant temperature. When the acoustic medium is in motion it is convenient to take the total or stagnation enthalpy B, rather than the pressure, as the fundamental acoustic variable (Howe 1975). In the present case this is defined by

$$
B=\int \frac{d p}{\rho}+\frac{l}{2} \stackrel{v}{ }^{2}
$$

Observe that in those regions where the flow is irrotational and can therefore be specified by a potential $\phi$, Bernoulli's equation implies that

$$
\frac{\partial \phi}{\partial t}=-B
$$

The equation which governs the generation of sound by distributions of vorticity has been derived by Howe (1975) who shows that for homentropic flow Lighthill's acoustic analogy theory may be expressed in terms of the inhomogeneous wave equation

$\left\{\frac{D}{D t}\left(\frac{I}{c^{2}} \frac{D}{D t}\right)+\frac{I}{c^{2}} \frac{D \underset{\sim}{D t}}{D t} \cdot \nabla-\nabla^{2}\right\} B=\operatorname{div}(\underset{\sim}{\omega}, \underset{\sim}{v})-\frac{I}{c^{2}} \frac{D \underset{\sim}{D t}}{D t} \cdot(\underset{\sim}{\omega}, \underset{\sim}{\mathrm{V}}), \quad(5.3)$

where $\underset{\sim}{\omega}=$ curl $\underset{\sim}{v}$ is the vorticity vector.

In order to apply this equation to the edge noise problem we shall introduce the following simplifying approximations. 
I. A boundary layer/wall jet turbulent eddy is frozen during the interval of time $\sim O(\delta / V)$ in which it convects past the trailing edge. This requires the lifetime $\delta / v$ to be large, i.e., $v \ll v$, and implies that $w$ is constant in a frame convecting at the local velocity $\mathrm{v}$. The "back reaction" of the trailing edge on the incident turbulence must therefore be negligible; this is more likely to be the case when induced vortex shedding occurs, which tends to smooth out large pressure gradients near the edge of the plate.

II. The eddy convection velocity $\underset{v}{v}$ is approximated by the local mean velocity $\underset{\sim}{V} \equiv \bigvee\left(x_{2}\right)$, a function of the perpendicular distance $x_{2}$ from the surface of the plate. At low Mach numbers the principal aerodynamic source term on the right of (5.3) and associated with the incident boundary layer/wall jet turbulence is therefore the dipole div $\left(\omega_{\wedge}, y\right) \simeq$ div $(\omega, V)$. This is expected to be an adequate approximation provĩdẽd that the turbulence differential scale is small compared with the length scale of the mean velocity $\underset{\sim}{V}$.

III. There is no significant correlation between incident turbulent eddies which translate at different mean convection velocities $v$.

IV. Effects of fluid compressibility are unimportant near the trailing edge. Terms in the wave operator of (5.3) which involve the sound speed $c$ are significant only in the propagation region, where fluctuations in $B$ may be linearized about a constant mean value $B_{0}$, say, and the velocity $\underline{v}$ set equal to $U_{0}$ in the positive $x_{1}$-direction.

V. The imposition of the Kutta condition results in the shedding of a distribution $\underset{\sim}{\omega}$ of vorticity into a wake which in the vicinity of the edge is approximated by a vortex sheet. The shed vorticity is frozen and convects at velocity $w=\left(w_{1}, 0, w_{3}\right)$.

VI. The mean shear velocity $V\left(x_{2}\right)$ and the wake convection velocity $W$ are both parallel to the unit vector $n$ and satisfy $M_{v}^{2}, M_{w}^{2} \ll 1$, where ${\underset{\sim}{W}}_{W}=\underset{\sim}{W} / c$.

Introducing these approximations into (5.3) we have:

$\left\{\frac{1}{c^{2}}\left(\frac{\partial}{\partial t}+U_{0} \frac{\partial}{\partial x_{1}}\right)^{2}-\nabla^{2}\right\} B=\operatorname{div}(\underset{\sim}{\omega} \underset{\sim}{V})+\operatorname{div}(\underset{\sim}{\widetilde{W}} \underset{\sim}{W})$, 
the terms on the right representing in turn the aeroacoustic sources associated with the incident and shed vorticity. These are dipoles with axes parallel respectively to $\underset{\sim}{\sim} \underset{\sim}{W}$ and $\tilde{\omega}_{\wedge} W$. Since $\bar{\omega}$ and $W$ must lie in the plane of the plate, the wake dipole is orientêd perpendicular to the plate in accordance with Hayden, Fox and Chanaud's (1976) hypothesis. The orientation of the incident boundary layer/wall jet vorticity $\underset{\sim}{\omega}$ is random, however, and so therefore is the corresponding dipole axis, although it will be shown that the direction of the principal component of this dipole is again perpendicular to the plane of the plate.

Let $Q$ denote the strength of the frozen incident dipole, i.e., set ,

$$
\underset{\sim}{\omega}\left(x_{1}-V_{1} t, x_{2}, x_{3}-V_{3} t\right) \wedge \underset{\sim}{V}\left(x_{2}\right)=Q\left(x_{1}-V_{1} t, x_{3}-V_{3} t, x_{2}\right) .
$$

The incident turbulence will be assumed to lie in $x_{2}>0$, so that we may also write:

$$
\underset{\sim}{Q} \equiv \int_{0}^{\infty} \underset{\sim}{Q}\left(x_{1}-V_{1} t, x_{3}-V_{3} t, z\right) \delta\left(x_{2}-z\right) d z,
$$

and this linear superposition reduces the problem of solving the inhomogeneous wave equation (5.4) to the consideration of a sequence of simpler aeroacoustic problems involving singular dipole sources of strength $Q \delta\left(x_{2}-z\right)$. The complete solution is obtained by integration over all $z$.

For each component $Q \delta\left(x_{2}-z\right)$ of the incident turbulent dipole there will be a corresponding component $\mathrm{q}_{\sim} \delta\left(\mathrm{x}_{2}\right)$ of the wake dipole $\underset{\sim}{\sim}, \underset{\sim}{W}$ such that

$$
\bar{\sim}_{\sim} \underset{\sim}{W}=\delta\left(x_{2}\right) \int_{0}^{\infty} \underset{\sim}{q}\left(x_{1}-W_{1} t, x_{3}-W_{3} t, z\right) d z,
$$

where the $\delta$-function signifies that the shed vorticity is confined to an infinitely thin wake downstream of the trailing edge. Thus Eq. (5.4) becomes

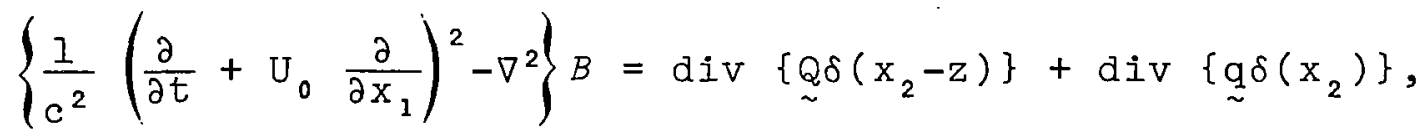


where

$$
\mathrm{B}=\int_{0}^{\infty} B \mathrm{dz}
$$

The unsteady flow induced by the dipole sources in (5.8) is irrotational in the vicinity of the rigid plate, and (5.2) therefore implies that the component $B$ satisfies the normal velocity condition $\partial B / \partial x_{2}=0$ on $\left(x_{2}=0, x_{1}<0\right)$. In addition $B$ must fulfill the radiation condition of outgoing acoustic waves at large distances from the edge of the plate. The techniques for solving the classical diffraction problem specified in this way are well documented (see, e.g., Noble 1958; Bowman, Senior and Uslenghi 1969) and we shall be content to quote the appropriate form of solution valid in the limit of small but finite mean flow Mach number, $M_{0}^{2} \ll 1$.

To do this, set

$$
B=B_{\mathrm{Q}}+B_{\mathrm{q}} \text {, }
$$

${ }_{Q}^{B}, B_{\mathrm{q}}$ corresponding respectively to the incident and wake dipole sources on the right of (5.8). We then have:

$$
\begin{aligned}
& B_{Q}=-\frac{1}{2} \int_{-\infty}^{\infty} \frac{\mu \cdot \hat{Q}}{\gamma\left(\mu_{1}\right)} e^{i\left\{\mu_{1} x_{1}+\gamma\left(\mu_{1}\right)\left(z-x_{2}\right)+\mu_{3} x_{3}-\omega_{z} t\right\}}
\end{aligned}
$$

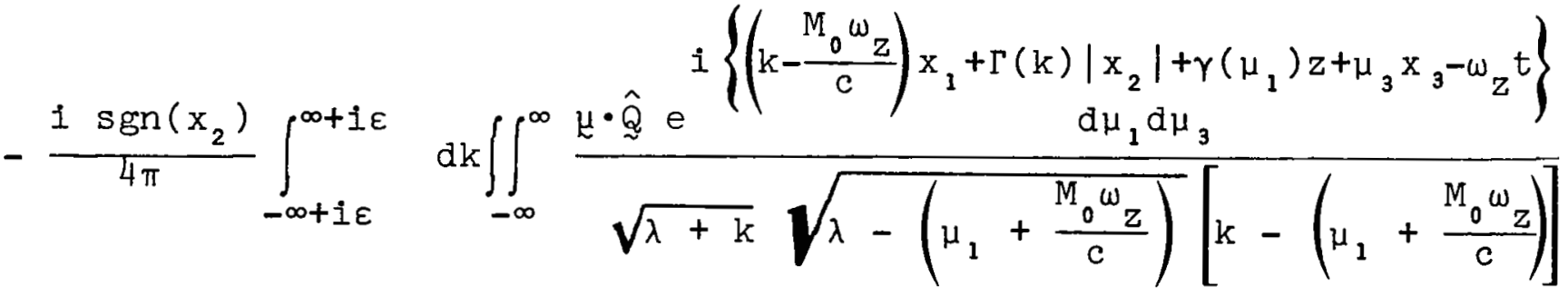

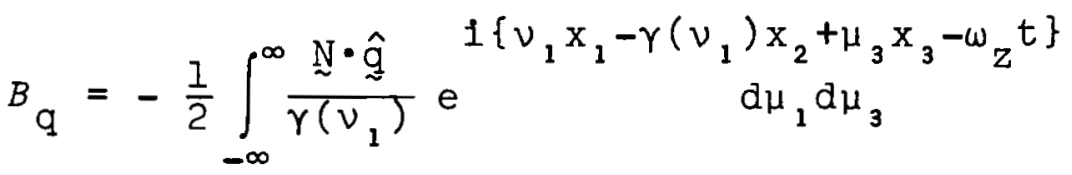

$-\frac{i \operatorname{sgn}\left(x_{2}\right)}{4 \pi} \int_{-\infty+1 \varepsilon}^{\infty+1 \varepsilon} d k \int_{-\infty}^{\infty} \frac{d \mu_{1} d \mu_{3} N \cdot \hat{q} e^{i\left\{\left(k-\frac{M_{0} \omega_{z}}{c}\right) x_{1}+\Gamma(k)\left|x_{2}\right|+\mu_{3} x_{3}-\omega_{z} t\right\}}}{\sqrt{\lambda+k} \sqrt{\lambda-\left(\nu_{1}+\frac{M_{0} \omega_{z}}{c}\right)}\left[k-\left(\nu_{1}+\frac{M_{0} \omega_{z}}{c}\right)\right]}$ 
where $\varepsilon \rightarrow+0$.

The various quantities appearing in these formulae have the following meanings. Fourier transforms $\hat{Q}$, $\hat{g}$ are defined typically by :

$\underset{\sim}{\hat{Q}} \equiv \underset{\sim}{\hat{Q}}\left(\mu_{1}, \mu_{3}, z\right)=\frac{1}{(2 \pi)^{2}} \int_{-\infty}^{\infty} \int_{\sim}^{Q}\left(x_{1}, x_{3}, z\right) e^{-1\left(\mu_{1} x_{1}+\mu_{3} x_{3}\right)} \underset{d x_{1} d x_{3}}{d}$.

The convective frequency $\omega_{z}$ is given by

$$
\omega_{z} \equiv \omega_{z}(z)=\underset{\sim}{\mu} \cdot \underset{\sim}{V}(z)=\mu_{2} V_{1}+\mu_{3} V_{3} ;
$$

after which we have

$$
\lambda=\left\{\begin{array}{l}
\operatorname{sgn}\left(\omega_{z}\right) \\
+i
\end{array}\left|\begin{array}{cc}
\frac{\omega_{z}^{2}}{c^{2}} & -\mu_{3}^{2}
\end{array}\right|^{1 / 2},\right.
$$

according as the argument of the radical is $+/-$, and similarly

$$
\begin{aligned}
& \gamma(\zeta)=\left\{\begin{array}{l}
\operatorname{sgn}\left(\omega_{z}\right) \\
+i
\end{array}\left|\lambda^{2}-\left(\zeta+\frac{M_{0} \omega_{z}}{c}\right)^{2}\right|^{1 / 2}\right. \\
& \Gamma(k)=\left\{\begin{array}{l}
\operatorname{sgn}\left(\omega_{z}\right) \\
+i
\end{array}\left|\lambda^{2}-k^{2}\right|^{1 / 2}\right.
\end{aligned}
$$

The three-dimensional vectors $\underset{\sim}{\mu}, \underset{\sim}{N}$ are given by

$$
\left.\begin{array}{l}
\underset{\sim}{\mu}=\left(\mu_{1},-\gamma\left(\mu_{1}\right), \mu_{3}\right) \\
\underset{\sim}{N}=\left(\nu_{1},-\gamma\left(\nu_{1}\right), \mu_{3}\right)
\end{array}\right\}
$$


where

$$
v_{1}=\frac{1}{W_{1}}\left\{\mu_{1} V_{1}+\mu_{3}\left(V_{3}-W_{3}\right)\right\}
$$

Finally those branches of $\sqrt{\lambda+k}, \sqrt{\lambda-\left(\mu_{1}+\frac{M_{0} \omega_{z}}{c}\right)}$,

$$
\sqrt{\lambda-\left(v_{1}+\frac{M_{0} \omega_{z}}{c}\right)}
$$

are chosen which are either positive or have positive imaginary parts on the real $\mathrm{k}, \mu_{1}, \nu_{1}$ axes respectively.

In the solutions (5.11), (5.12) the first of the integrals on the right hand side of each expression represents respectively the evanescent wave field that would be generated by the dipoles $Q$, $q$ in the absence of the plate, and are valid at the plate for $\tilde{x}_{2} \leq 0$ only. The multiple integrals account for diffraction by the plate and are valid for arbitrary $x_{2}$. In writing down these results recall that the dipole strength $Q$ is defined by the frozen pattern of vorticity in the turbulent boundary layer/wall jet. The strength $q$ of the wake dipole is unknown, however, and can be determined önly when the precise conditions at the trailing edge are specified. We shall examine the opposite extremes in which either no vortex shedding occurs at the edge, or the full Kutta condition is imposed and vortex shedding is permitted in order to remove the trailing edge potential flow singularity. In the first case $q \equiv Q$. On the other hand, when the Kutta condition is applied the flow must leave the edge tangentially, and it follows from (5.2) that

$$
\frac{\partial B}{\partial x_{2}} \rightarrow 0 \text { as } x_{1} \rightarrow+0 \text { on } x_{2}=0
$$

In order to calculate $g$ from this condition observe that only the multiple-integra $\tilde{l}$ contributions to ${ }^{B}{ }$, ${ }^{B}$ in $(5.11)$, (5.12) are singular, and therefore for small positive $\mathrm{x}_{1}$ on $\mathrm{x}_{2}=$ 0 , we have 


$$
\begin{aligned}
& \frac{\partial B}{\partial \mathrm{x}_{2}} \equiv \frac{\partial}{\partial \mathrm{x}_{2}}\left\{B_{\mathrm{Q}}+B_{\mathrm{q}}\right\} \\
& =\frac{1}{4 \pi} \int_{-\infty+1 \varepsilon}^{\infty+i \varepsilon} d k \iint_{-\infty}^{\infty} d \mu_{1} d \mu_{3} \sqrt{\lambda-k}
\end{aligned}
$$

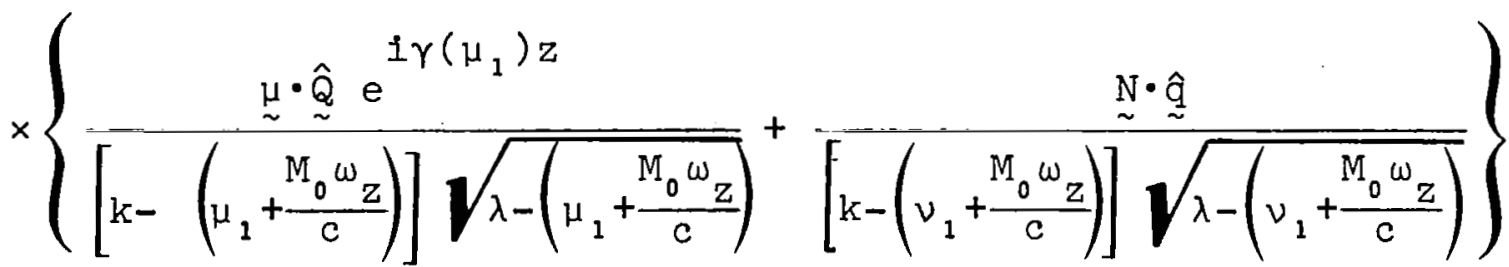

$$
\begin{aligned}
& x e^{i\left[\left(k-\frac{M_{0} \omega}{c}\right) x_{1}+\mu_{3} x_{3}-\omega_{z} t\right]} .
\end{aligned}
$$

For arbitrary $\hat{\mathrm{q}}$ the non-exponential term in the integrand $\sim 0(1 / \sqrt{k})$ as $k \rightarrow \infty \sim$, and this implies that $\partial B / \partial x_{2} \sim 0\left(1 / \sqrt{x_{1}}\right)$ as $x_{1}$ $\rightarrow+0$ (see, e.g., Noble 1958). By means of the choice

$$
\underset{\sim}{N} \cdot \underset{\sim}{\hat{q}}=-\underset{\sim}{\mu} \cdot \underset{\sim}{Q} e^{i \gamma\left(\mu_{1}\right) z} \sqrt{\frac{\lambda-\left(\nu_{1}+\frac{M_{0} \omega_{z}}{c}\right)}{\lambda-\left(\mu_{1}+\frac{M_{0} \omega_{z}}{c}\right)}}
$$

the term in the curly brackets of the integrand tends to zero at least as fast as $k^{-2}$ as $k \rightarrow \infty$, and this is sufficient to ensure that $\partial B / \partial x_{2} \rightarrow 0$ at the edge. For each $z$ the disturbed flow on either side of the vortex sheet wake is irrotational and Eq. (5.20) together with the definition (5.7) of $\mathrm{g}$ may be used to determine the strength $\bar{w}$ of the shed vorticity, although this calculation is not required in the present discussion. 
Equation (5.20) leads to an important conclusion, which generalizes an earlier two-dimensional result noted by Howe (1976), that no edge noise is generated in the particular case in which the incident and shed vorticity convect at the same mean velocity. To see this observe that if $W=V(z),(5.18)$ implies that $\nu_{1} \equiv \mu_{1}$, and therefore when $\hat{q}$ is chosen to satisfy. (5.20), the integrand of $(5.19)$ is identĩcally zero for arbitrary values of $k$. Thus the diffractional component of $B=B_{0}+B$ vanishes. When this happens the field due to the shed vorticity is exactly that which would be produced by an image of the incident dipole $Q$ in an infinite rigid plate, the normal derivative $\partial B / \partial x_{2}$ being zero at all points of the plate and wake. Under these circumstances there is no acoustic radiation from the uniformly convecting frozen turbulence and its image in $x_{2}=0$, because at subsonic convection velocities the first terms on the right of each of (5.11), (5.12) decay more rapidly than $1 / R$ in the far field.

\section{SECTION 5.2 The Far Field Sound}

The far field acoustic radiation is obtained by evaluating the multiple integrals of each of (5.11), (512) by the method of steepest descents (Watson 1966 page 235), the saddle point in the $\left(k, \mu_{3}\right)$-plane being located at

$$
k=\frac{\omega_{z}}{c} \sin \alpha \cos \bar{\theta} ; \quad \mu_{3}=\frac{\omega_{z}}{c} \cos \alpha,
$$

where $(R, \bar{\theta}, \alpha)$ are the observer coordinates defined in Sec. 2 . The remaining integration with respect to $\mu_{1}$ may be expressed as an integral over all values of the convective frequency $\omega_{z}$ by means of ( 5.14 ) and the suffix $z$ is accordingly discarded. "Thus we obtain the following far field representation of $B_{Q}$ :

$$
\begin{aligned}
B_{Q} & =\frac{-i \sqrt{M_{V 1}} \sin \left(\frac{\bar{\theta}}{2}\right) \sqrt{\sin \alpha}}{R \sqrt{2}\left(1-M_{V R}\right)\left(1-M_{V_{1}} \sin \alpha-M_{V_{3}} \cos \alpha\right)^{1 / 2}} \\
& \times \int_{-\infty}^{\infty} \frac{d \omega}{\omega}[\underset{\sim}{\mu} \cdot \underset{\sim}{\hat{Q}}] \exp \left\{-\frac{|\omega| z}{V_{1}}\left(1-M_{V_{3}} \cos \alpha\right)+\frac{i \omega}{c}\left(R-M_{0} x_{1}-c t\right)\right\} \cdot(5.22)
\end{aligned}
$$



In this formula the notation $[\underset{\sim}{\mu} \cdot \underset{\sim}{\hat{Q}}]$ indicates that $\underset{\sim}{\mu} \cdot \hat{\sim}\left(\mu_{1}, \mu_{3}, z\right)$ is
to be evaluated at

$$
\begin{aligned}
& \mu_{1}=\frac{\omega}{V_{1}}\left(I-M_{v_{3}} \cos \alpha\right) \\
& \mu_{2}=-i \frac{|\omega|}{V_{1}}\left(I-M_{v_{3}} \cos \alpha\right) \\
& \mu_{3}=\frac{\omega}{c} \cos \alpha
\end{aligned}
$$

and $M_{V R}$ denotes the component of the convection Mach number $\underset{\sim}{M}$ in the observer direction.

To interpret $(5.22)$ it is necessary to express $B_{0}$ in terms of the corresponding acoustic perturbation pressure $\mathrm{p}_{Q}$. Using Eqs. (5.1), (5.2) in the far field it is readily shown that, at small mean flow Mach numbers, $M_{0}$,

$$
p_{Q}=\frac{\rho_{0}^{B} Q}{\left(1+M_{O R}\right)}
$$

where $M_{O R}=M_{0}\left(x_{1} / R\right)$ is the component of $\left(M_{0}, 0,0\right)$ in the observer direction. The total acoustic pressure perturbation produced by the turbulent boundary layer/wall jet in the absence of vortex shedding $p_{I}$, say, is now obtained by integration with respect to $z$, as in Eq. (5.9). This yields:

$p_{I}=\frac{-i \rho_{0} \sin \left(\frac{\bar{\theta}}{2}\right) \sqrt{\sin \alpha}}{R \sqrt{2}\left(I+M_{O R}\right)} \int_{-\infty}^{\infty} \frac{d \omega}{\omega} \int_{0}^{\infty} d z$

$$
\times \frac{\sqrt{M_{v_{1}}}[\underset{\sim}{\mu} \cdot \hat{Q}] \exp \left\{-\frac{|\omega| z}{V_{1}}\left(1-M_{v_{3}} \cos \alpha\right)+\frac{i \omega}{c}\left(R-M_{0} x_{1}-c t\right)\right\}}{\left(I-M_{v_{R}}\right)\left(I-M_{v_{1}} \sin \alpha-M_{v_{3}} \cos \alpha\right)^{1 / 2}}
$$


Similarly, adding Eqs. (5.11), (5.12) and using (5.20) we obtain the following expression for the acoustic pressure $p_{K}$ in the case in which the Kutta condition is enforced:

$$
\begin{aligned}
& p_{K}= \frac{-i \rho_{0} \sin \left(\frac{\bar{\theta}}{2}\right) \sqrt{\sin \alpha}}{R \sqrt{2}\left(1+M_{O R}\right)\left(1-M_{W R}\right)} \int_{-\infty}^{\infty} \frac{d \omega}{\omega} \int_{0}^{\infty} d z \\
& \times \frac{\sqrt{M_{v_{1}}}(1-\sigma(z))[\underset{\sim}{\mu} \cdot \underset{\sim}{\hat{Q}}] \exp \left\{-\frac{|\omega| z}{V_{1}}\left(1-M_{v_{3}} \cos \alpha\right)+\frac{i \omega}{c}\left(R-M_{0} x_{1}-c t\right)\right\}}{\left(1-M_{V R}\right)\left(1-M_{v_{1}} \sin \alpha-M_{v_{3}} \cos \alpha\right)^{1 / 2}}
\end{aligned}
$$
$M_{W R}$ being the component of the wake convection Mach number ${ }_{\sim}{ }_{W}$ in
the observer direction. The function

$$
\sigma(z)=W / V(z)
$$

is the ratio of the wake convection velocity to that in the boundary layer/wall jet flow at a distance $z$ from the surface of the plate. Note in particular that the component of $\mathrm{p}_{\mathrm{K}}$ at which $\sigma=1$ is identically zero, the sound produced by the corresponding incident and shed frozen vorticity distributions being equal and opposite.

SECTION 5.3 The Principal Edge-Noise Dipole

Consider now the particular case in which the component of the mean boundary layer/wall jet flow parallel to the edge of the plate is small, i.e., $\tan \beta=\mathrm{v}_{3} / \mathrm{V}_{1}=\mathrm{w}_{3} / \mathrm{w}_{1} \ll 1$. 
Let $\hat{\omega}=\left(\hat{\omega}_{1}, \hat{\omega}_{2}, \hat{\omega}_{3}\right)$ denote the Fourier transform of the incident vorticity $\underset{\sim}{\omega}$ defined as in (5.13), then

$$
[\underset{\sim}{\mu} \cdot \hat{Q}] \equiv\left[\underset{\sim}{\mu} \cdot{\underset{\sim}{\omega}}_{\sim} \underset{\sim}{V}(z)\right] \simeq-i \hat{\omega}_{3}|\omega|
$$

at low mean flow Mach numbers. This shows that in the present circumstances the leading contribution to the edge noise is provided by the component of vorticity parallel to the edge of the plate, the corresponding acoustic dipole $Q=w_{\sim} V$ being perpendicular to the plane of the plate.

In general the wetted span $L$ of the edge greatly exceeds the turbulence correlation scale, and it may therefore be assumed that within the turbulent flow, and in the neighborhood of the edge where the turbulence may be regarded as frozen, the vorticity $\omega$ constitutes a stationary random function of the coordinates $\left(x_{1}, x_{3}\right)$ parallel to the plate. For the moment we shall also suppose that the turbulence convection velocity $V$ is independent of the distance $z$ from the surface of the plate.

means of

A vorticity spectrum density $\Phi_{33}$ may now be introduced by

$$
\begin{aligned}
\left\langle\hat{\omega}_{3}\left(\mu_{1}, \mu_{3}, z\right) \hat{\omega}_{3}\left(\bar{\mu}_{1}, \bar{\mu}_{3}, \bar{z}\right)\right\rangle & =v^{2}\left(\frac{l_{1} \ell_{3}}{\ell_{0} \ell_{2}}\right) \Phi_{33}\left(\ell_{1} \mu_{1}, \ell_{3} \mu_{3}, \frac{\bar{z}-z}{\ell_{2}}, \frac{z}{\ell_{0}}\right) \delta\left(\mu_{1}+\bar{\mu}_{1}\right) \\
& \times \frac{\sin \left[\frac{\left.L_{2}\left(\mu_{3}+\bar{\mu}_{3}\right)\right]}{\pi\left(\mu_{3}+\bar{\mu}_{3}\right)} .\right.}{(5.29)}
\end{aligned}
$$

In this definition, $\ell_{1}, \ell_{2}, \ell_{3}$ are respectively vorticity correlation scales in the $(1,2,3)$-directions, and $l_{0}$ represents the length scale of the profile of the mean boundary layer/wall jet. The function $\Phi_{33}$ is dimensionless, and for points $\left(x_{1}, z_{2}, x_{3}\right)$, $\left(X_{1}, z, x_{3}\right)$ lying within the turbulent flow the vorticity correlation is given by:

$$
\begin{aligned}
&\left.<\omega_{3}\left(x_{1}, x_{3}, z\right) \omega_{3}\left(x_{1}, X_{3}, \bar{z}\right)\right\rangle \equiv\left(\frac{v^{2}}{\ell_{0} l_{2}}\right) \iint_{-\infty}^{\infty} \Phi_{33}\left(l_{1} \mu_{1}, \ell_{3} \mu_{3}, \frac{\bar{z}-z}{l_{2}}, \frac{z}{l_{0}}\right) \\
& \times e^{i\left\{\mu_{1}\left(x_{1}-X_{1}\right)+\mu_{3}\left(x_{3}-X_{3}\right)\right\}} \\
& d\left(l_{1} \mu_{1}\right) d\left(l_{3} \mu_{3}\right) .
\end{aligned}
$$


Equation ( 5.29 ) may be used to calculate the leading contribution to the acoustic spectrum $S_{I}(\omega)$ (defined as in (3.4)) in the approximation of (5.28). In the absence of a Kutta condition we have from (5.25):

$$
\begin{aligned}
S_{I}(\omega) & =\frac{\rho_{0}^{2} v^{2} V^{2} M_{V}}{2 \pi}\left(\frac{L l_{3}}{R^{2}}\right) \frac{\sin \alpha \cdot \sin ^{2}\left(\frac{\bar{\theta}}{2}\right) \cos ^{3} \beta}{\left(I+M_{O R}\right)^{2}\left(1-M_{V R}\right)^{2}\left(1-M_{V} \sin \alpha\right)} \\
& \times\left(\frac{l_{1}}{V_{1}}\right) \times\left(\frac{\omega l_{1}}{V_{1}}, 0\right)
\end{aligned}
$$

in which $V$ is constant, and the dimensionless spectral density $X$ is given $\tilde{b} y$

$$
\begin{aligned}
\chi\left(\ell_{1} \mu_{1}, l_{3} \mu_{3}\right) & =\frac{1}{\ell_{0} l_{2}} \iint_{0}^{\infty} \mathrm{dzd} \bar{z} \Phi_{33}\left(\ell_{1} \mu_{1}, \ell_{3} \mu_{3}, \frac{\bar{z}-z}{\ell_{2}}, \frac{z}{\ell_{0}}\right) \\
& \times e^{\frac{-\left|\mu_{1}\right| l_{1}}{V_{1}}(z+\bar{z})},
\end{aligned}
$$

and depends on the structure of the boundary layer/wall jet profile.

The result (5.31) gives the distribution of acoustic power as a function of the strouhal number $\omega l_{1} / \mathrm{v}_{1}$ based on the eddy length scale and convection velocity parallel to the plate and perpendicular to the edge. Let $c_{x}$ be the numerical coefficient defined by:

$$
c_{x}=\int_{0}^{\infty} x\left(\frac{\omega \ell_{1}}{v_{1}}, 0\right) d\left(\frac{\omega \ell_{1}}{v_{1}}\right),
$$


then the OASPL predicted by (5.31) becomes:

$$
\left\langle p_{I}^{2}\right\rangle=\frac{{ }^{c} \rho_{0}^{2} v^{2} V^{2} M_{v}}{2 \pi}\left(\frac{L l_{3}}{R^{2}}\right) \frac{\sin \alpha \sin ^{2}\left(\frac{\bar{\theta}}{2}\right) \cos ^{3} \beta}{\left(1+M_{O R}\right)^{2}\left(1-M_{V R}\right)^{2}\left(I-M_{V_{1}} \sin \alpha\right)} .
$$

This result reduces to the basic ffowcs Williams-Hall prediction formula (2.7) when the Doppler factors associated with the ambient mean flow and source convection are discarded.

A more convenient representation of the effect of forward flight is in terms of the coordinates of the far field observer relative to the edge of the plate at the time of emission of the sound. The observer is assumed to be fixed relative to the ground, i.e., relative to the ambient mean flow $\left(U_{0}, 0,0\right)$, corresponding to flight at speed $U_{0}$ in the negative $x_{1}$-direction. Let $(r, \Theta, \Phi)$ be the spherical polar coordinates of the observer at the emission time, $\theta$ being measured from the positive $x_{1}$-axis. The appropriate transformation of coordinates is given by

$$
\left.\begin{array}{l}
x_{1}=r\left(M_{0}+\cos \theta\right) \\
x_{2}=r \cos \Phi \cdot \sin \theta \\
x_{3}=r \sin \Phi \cdot \sin \theta
\end{array}\right\} .
$$

and is illustrated in the "flyover"-plane $(\Phi=0)$ in Fig. 5 . These formulae may be used to express the observer dependent quantities $(R, \bar{\theta}, \alpha), M_{O R}, M_{Y R}$ in $(5.34)$ in terms of the emission time coordinates. The resulting expression for the sound pressure level assumes a simplified form in the flyover plane, for which we find:

$$
\left\langle p_{I}^{2}\right\rangle=\frac{c \gamma \rho_{0}^{2} v^{2} V^{2} M_{V} v}{2 \pi}\left(\frac{L \ell_{3}}{r^{2}}\right) \frac{\left(I-M_{0}+M_{v_{1}}\right) \sin ^{2}\left(\frac{\theta}{2}\right) \cos ^{3} \beta}{\left(1+M_{0} \cos \theta\right)^{3}\left\{I+\left(M_{0}-M_{v_{1}}\right) \cos \theta\right\}^{2}} \cdot \quad \text { (5.36) }
$$




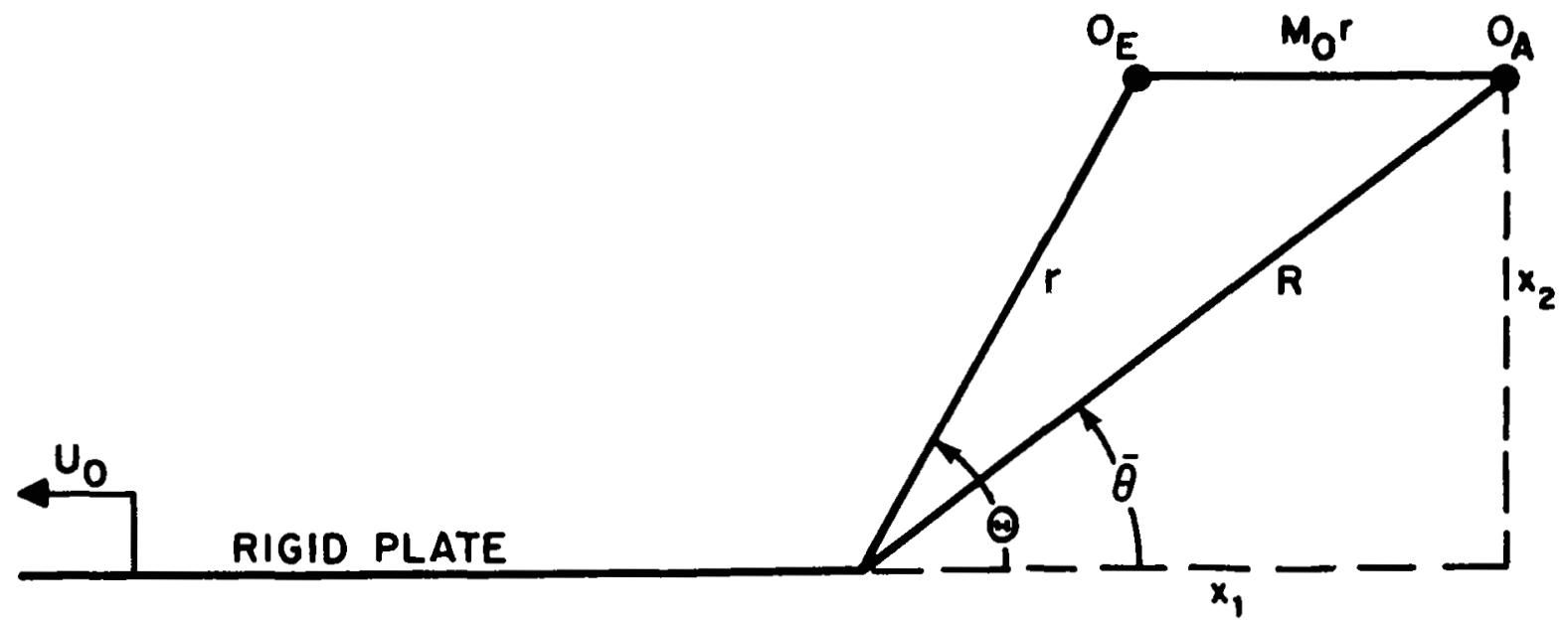

FIG. $50_{E}(r, \theta)$ IS THE LOCATION IN THE FLYOVER-PLANE OF THE GROUND-FIXED OBSERVER RELATIVE TO THE EDGE OF THE PLATE AT THE TIME OF EMISSION OF THE SOUND; $0_{A}(R, \bar{\theta})$ IS THE RELATIVE LOCATION AT THE TIME OF ARRIVAL OF THE SOUND. 
Similarly it may be deduced from (5.26) that in the approximation of small $\beta$, when the Kutta condition is imposed the OASPL is given by:

$$
\begin{aligned}
& \left\langle p_{K}^{2}\right\rangle=\frac{c_{X} \rho_{0}^{2} v^{2} V^{2} M_{V}(I-\sigma)^{2}}{2 \pi}\left(\frac{L_{l_{3}}}{r^{2}}\right) \\
& \left(1-M_{0}+M_{v_{1}}\right) \sin ^{2}\left(\frac{\Theta}{2}\right) \cos ^{3} \beta \\
& \left(1+M_{0} \cos \theta\right)\left[I+\left(M_{0}-M_{W_{1}}\right) \cos \theta\right]^{2}\left[I+\left(M_{0}-M_{V_{1}}\right) \cos \theta\right]^{2} \text {, }
\end{aligned}
$$

where $M_{W 1}=W_{1 / c}$ and $\sigma=W / V$.

Comparison of $(5.36)$ and $(5.37)$ reveals that:

(a) Application of the Kutta conditign reduces the predicted OASPL by a factor of $(1-\sigma)^{2} \equiv(V-W)^{2} / V^{2}$.

(b) Increasing the flight Mach number $M_{0}$ reduces the SPL at $\theta=90^{\circ}$ because of the relative velocity factor $\left(1-M_{0}+M_{v 1}\right)$. Assuming that all velocities vary in proportion, this suggests that at higher Mach numbers (e.g., 0.5, say), the SPL will tend to fall below the $U^{5}$ datum of the Ffowcs Williams \& Hall (1970) theory. There may also be additional departures from the fifth power law arising from possible dependencies of the wetting length $L$ and the transverse correlation scale $\ell_{3}$ on velocity. It should be noted that, although terms $0\left(h^{2}\right)$ have been systematically neglected relative to unity in obtaining the above results, the Mach number dependencies of the final formulae $(5.36),(5.37)$ are in precise agreement with those expected from an exact analysis (see Howe 1977).

(c) Forward flight and source motion effects are characterized by five Doppler factors. In the No-Kutta condition case two are associated with motion of the boundary layer/wall jet sources relative to the observer, and three with the flight velocity $U_{0}$. When the Kutta condition is imposed only one Doppler factor arises from the flight velocity, but there are two each from the incident and shed vortical dipole sources. 
The order of magnitude of the differences in the sound pressure levels predicted by (5.36) and (5.37) may be estimated in the case of an airfoil, for which we assume that close to the trailing edge the eddy convection Mach number in the upper turbulent boundary layer is given by:

$$
\mathrm{M}_{\mathrm{v}_{1}} \simeq 0.7 \mathrm{M}_{0} \text {, }
$$

and in the near wake

$$
\mathrm{M}_{\mathrm{W}_{1}} \simeq 0.5 \mathrm{M}_{0} \text {. }
$$

Let $\triangle$ SPL dB denote the amount by which the No-Kutta-condition prediction (5.36) exceeds the Kutta condition prediction (5.37), i.e.,

$$
\Delta S P I=10 \log _{10}\left\{\frac{1}{(1-\sigma)^{2}}\left(\frac{1+\left(M_{0}-M_{W_{1}}\right) \cos \theta}{1+M_{0} \cos \theta}\right)^{2}\right\} .
$$

At a flight Mach number of $M_{0}=0.3$ the variation of $\triangle S P L$ with the flyover angle $\theta$ is given in Table 1.

TABLE 1. TYPICAL VARIATION WITH FLYOVER ANGLE OF $\triangle S P L=$ NO-KUTTACONDITION SPL/KUTTA-CONDITION SPL

\begin{tabular}{|c|c|c|c|c|c|c|c|}
\hline$\theta$ & $0^{\circ}$ & $30^{\circ}$ & $60^{\circ}$ & $90^{\circ}$ & $120^{\circ}$ & $150^{\circ}$ & $180^{\circ}$ \\
\hline$\triangle \mathrm{SPL}(\mathrm{dB})$ & 9.8 & 9.9 & 10.3 & 10.9 & 11.6 & 12.3 & 12.6 \\
\hline
\end{tabular}


There is evidently a significant difference in the predicted sound pressure levels over the whole range of directions, although the variation due to Doppler factor differences is small, amounting to no more than $3 \mathrm{~dB}$. Such large overall differences are an indication of the weakness of the present state of the theory. Ignorance of the precise condition to be applied at the trailing edge can apparently result in an uncertainty in the predicted noise levels which is of the order of $10 \mathrm{~dB}$. The same difficulty arises when the edge noise problem is formulated in terms of the pressure fluctuations at or near the trailing edge, to which we now turn our attention.

\section{SECTION 5.4 Relation Between Surface Pressure Fluctuations and the Far Field Sound}

Consider the general case specified by the formal solutions (5.11), (5.12), with $N \cdot \hat{g}$ given by (5.20). We use these results to determine the fluctuating pressure on the surface of the plate.

On the rigid plate the result corresponding to (5.24) which relates $\mathrm{p}_{\mathrm{Q}}$ and ${ }^{B} \mathrm{Q}$ becomes

$$
p_{Q}=\frac{\rho_{0}}{\omega}(\omega+\underset{\sim}{i} \cdot \nabla) B_{Q}
$$

for each harmonic component of frequency $\omega$. This is used in conjunction with $(5.11),(5.12)$ to express the surface pressure in the form

$$
\begin{aligned}
& p_{K}=-\frac{\rho_{0}}{2} \iint_{-\infty}^{\infty} d \mu_{1} d \mu_{3} \int_{0}^{\infty} d z\left\{1+\operatorname{sgn}\left(x_{2}\right) \operatorname{Erf}\left(\sqrt{i x_{1}\left[\lambda+\mu_{1}+\frac{\bar{M}_{0} \omega_{z}}{c}\right]}\right)\right. \\
& \times \frac{\underset{\sim}{\mu \cdot(V-W)(\underset{\sim}{\mu} \cdot \hat{Q})}}{\omega_{z} \gamma\left(\mu_{1}\right)} e^{i\left\{\mu_{1} x_{1}+\mu_{3} x_{3}+z \gamma\left(\mu_{1}\right)-\omega_{z} t\right\}}
\end{aligned}
$$


when the Kutta condition is imposed (c.f. Chase 1975). In this expression the error function characterizes the diffraction by the trailing edge, and $\operatorname{sgn}\left(x_{2}\right)=+/$-according as the rigid surface is approached from positive or negative values of $x_{2}$. As $x_{1} \rightarrow-\infty$ on the upper surface $\left(x_{2}=+0\right)$ (5.40) predicts the familiar pressure doubling of the pseudo-sound pressure fluctuations. On the lower surface $\left(x_{2}=-0\right)$ the pressure fluctuations tend rapidly to zero upstream of the trailing edge. The pressure fluctuations in the wake $\left(x_{2}=0, x_{1}>0\right)$ are also given by (5.40) when the contribution from the error function is discarded and provided that the mean shear in the wake is small.

Chase (1975) observed that the presence of the error function in (5.40) implies that only at points close to the edge of the plate can a simple relation be expected to exist between surface pressure fluctuations and those in the acoustic far field. In order to neglect the contribution from the error function attention must be confined to points $x_{1}$ located either in the wake, or on the plate well within a characteristic hydrodynamic wavelength of the edge. It may then be assumed that the pressure fluctuations induced by the frozen vorticity are a stationary random function of $\left(x_{1}, x_{3}, t\right)$. In this region a pressure spectrum function $\pi_{K}\left(\mu_{1}, \mu_{3}, \sigma\right)$ is introduced which satisfies:

$$
\begin{aligned}
<p_{K}\left(x_{1}, x_{3}, t\right) p_{K}\left(x_{1}+x_{1}, x_{3}+X_{3}, t+\tau\right)> & =\int_{-\infty}^{\infty} \Pi_{K}\left(\mu_{1}, \mu_{3}, \omega\right) \\
& \times e^{i\left(\mu_{1} x_{1}+\mu_{3} x_{3}-\omega \tau\right)} d \mu_{1} d \mu_{3} d \omega .
\end{aligned}
$$

The neglect of the diffractional error function term in (5.40) implies that the corresponding wavenumber-frequency spectrum $\Pi_{K}\left(\mu_{1}, \mu_{3}, w\right)$ coincides with the spectrum of the pseudo-sound pressure fluctuations considered by Chandiramani (1974) and Chase (1975). Those fluctuations are produced by harmonic turbulent elements of wavenumber $\left(\mu_{1}, \mu_{3}\right)$ and frequency $\omega_{z}$ convecting at velocity $\underset{\sim}{ } \underset{\sim}{ }\left(\omega_{z} / \underset{\sim}{\mu} \cdot \underset{z}{n}\right)$, $n$ being the unit vector parallel to $\mathrm{V}$ introduced in Sec. $\tilde{3}$, and their statistical properties are determined by those of the incident voriticty distribution contained in the component $\underset{\sim}{\mu} \cdot \hat{Q}$ of the integrand of $(5.40)$. 
By analogy with (5.29) above a vorticity spectrum function i may be defined by means of:

$$
\begin{aligned}
\left\langle\underset{\sim}{\mu} \cdot \hat{Q}\left(\mu_{1}, \mu_{3}, z\right)\right\}\left\{\underset{\sim}{\mu} \cdot \hat{Q}\left(\bar{\mu}_{1}, \bar{\mu}_{3}, \bar{z}\right)\right\}> & =-\Phi_{i}\left(\ell_{1} \mu_{1}, \ell_{3} \mu_{3}, \frac{\bar{z}-z}{\ell_{2}} \frac{z}{\ell_{0}}\right) \\
& \times \delta\left(\mu_{1}+\bar{\mu}_{1}\right) \frac{\sin \left[\frac{L}{2}\left(\mu_{3}+\mu_{3}\right)\right]}{\pi\left(\mu_{3}+\bar{\mu}_{3}\right)},(5.42)
\end{aligned}
$$

where $\Phi_{i} \geq 0$, in which case we have from (5.41):

$$
\begin{aligned}
& \left\langle p_{K}\left(x_{1}, x_{3}, t\right) p_{K}\left(x_{1}+x_{1}, x_{3}+X_{3}, t+\tau\right)>=\frac{\rho}{4} \iint_{-\infty}^{\infty} d \mu_{1} d \mu_{3} \iint_{0}^{\infty} \frac{[l-\sigma(z)]^{2}}{\left|\gamma\left(\mu_{1}\right)\right|^{2}}\right. \\
& \times \Phi_{i}\left(\ell_{1} \mu_{1}, \ell_{3} \mu_{3}, \frac{\bar{z}-z}{\ell_{2}}, \frac{z}{\ell_{0}}\right) e^{-2 z\left|\gamma\left(\mu_{1}\right)\right|+i\left\{\mu_{1} X_{1}+\mu_{3} X_{3}-\mu \cdot V(z) \tau\right\}}
\end{aligned}
$$

where $\mu \cdot V(z)$ has been substituted for $\omega_{z}$. In writing down this result ${ }^{\sim}$ we have made use of the assumption that a significant correlation can exist between turbulent eddies only if they have the same convection velocity $V(z)=V(\bar{z})$. Set:

$f\left(\mu_{1}, \mu_{3}, z\right)=\frac{\rho_{0}^{2}[1-\sigma(z)]^{2}}{4\left|\gamma\left(\mu_{1}\right)\right|^{2}} e^{-2 z\left|\gamma\left(\mu_{1}\right)\right|} \int_{0}^{\infty} \Phi_{i}\left(l_{1} \mu_{1}, \ell_{3} \mu_{3}, \frac{\bar{z}-z}{l_{2}}, \frac{z}{l_{0}}\right) d \bar{z}$ 
which defines a wavenumber spectrum $f\left(\mu_{1}, \mu_{3}, z\right)$ whose magnitude is proportional to the mean square pseudo-sound pressure fluctuations near the edge produced by an eddy of wavenumber $\left(\mu_{1}, \mu_{3}\right)$ located in a plane parallel to the plate and distant $z$ from the surface.

It follows from (5.41) and (5.43) that the wavenumber-frequency spectrum $\Pi_{K}$ of pressure fluctuations in the wake and on the plate close to the trailing edge is given by:

$$
\Pi_{K}\left(\mu_{1}, \mu_{3}, \omega\right)=\int_{0}^{\infty} f\left(\mu_{1}, \mu_{3}, z\right) \delta(\omega-\underset{\sim}{\mu} \cdot V) d z .
$$

This represents $\Pi_{K}$ as a sum of independent contributions from different levels $z$ in the turbulent boundary layer/wall jet.

Consider next the acoustic far field. Let $S_{K}(\omega)$ denote the pressure spectral density defined as in (3.4). The general solution (5.26) may be used to express $S_{K}(\omega)$ in terms of $f$, as follows:

$S_{K}(\omega)=\frac{2 L}{\pi c R^{2}} \frac{\sin \alpha \cdot \sin ^{2}\left(\frac{\bar{\theta}}{2}\right)}{\left(I+M_{O R}\right)^{2}\left(I-M_{W R}\right)^{2}} \int_{0}^{\infty} \frac{f\left(\frac{\omega}{V_{1}}\left(1-M_{v_{3}} \cos \alpha\right), \frac{\omega}{c} \cos \alpha, z\right) d z}{\left(1-M_{V R}\right)^{2}\left(1-M_{V} \sin \alpha\right)}$.

Taking advantage of the $\delta$-function in (5.45) and using the transformation

$$
\mu_{1}=\frac{\omega}{V_{1}(z)}\left(I-M_{V_{3}} \cos \alpha\right)
$$


to express (5.46) as an integral (or set of integrals) with respect to $\mu_{1}$, it is easy to see that $f\left(\mu_{1}, \mu_{3}, \omega\right)$ can be eliminated between $(5.45),(5.46)$ to yield the following relation between the acoustic spectrum and the pseudo-sound wavenumber-frequency spectrum:

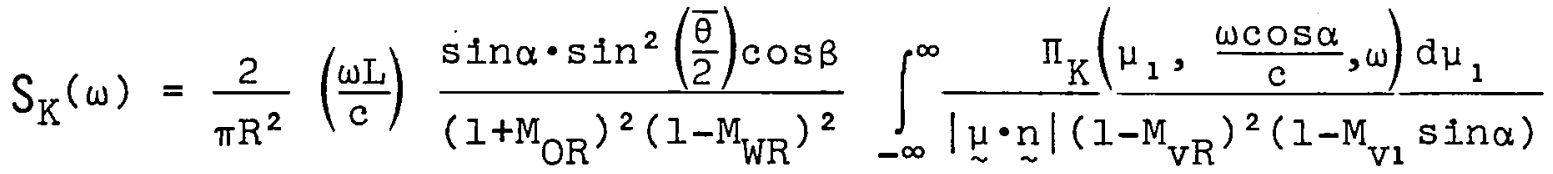

in which $\mu=\left(\mu_{1}, \frac{\omega \cos \alpha}{c}\right), M_{v R}$ is the component of the eddy convection Mach number $(\omega / c \mu \cdot n)$ n in the observer direction, and $M_{y 1}=\left(\omega / c_{\mu} \cdot n\right) \cos \beta$. This is the gẽneral form of the result (3.5) given by Ch̆as̃e (1975).

In practice (5.47) is likely to be of limited utility. This is because the assumption that pressure fluctuations in the wake are given by the incident evanescent wave spectrum $\Pi_{K}\left(\mu_{1}, \mu_{3}, \omega\right)$ is an approximation which is valid only when the shear layer interaction term $v_{2} \partial \mathrm{V} / \partial \mathrm{x}_{2}$ is negligible. This is the condition for (5.39) - which is strictly correct on the rigid surface of the plate - to continue to be valid in the wake. Thus reliable estimates of $\Pi_{K}$ must be based on surface pressure measurements alone, a proposition which is incompatible with the condition that those measurements be restricted to positions $x_{1}$ closer than a characteristic eddy length scale to the edge of the plate. This difficulty is absent when the eddy convection velocity $V$ is effectively constant and equal to $\bar{V}$, say. In this case $|\mu \cdot \underset{\sim}{n}|(\simeq \omega / \bar{V})$ and the Doppler factors may be taken outside the intẽgral sign in $(5.47)$ to give:

$$
S_{K}(\omega) \simeq \frac{2 M_{\bar{v}} I \sin \alpha \sin ^{2}\left(\frac{\bar{\theta}}{2}\right) \cos \beta \int_{-\infty}^{\infty} \Pi_{K}\left(\mu_{1}, \frac{\omega \cos \alpha}{c}, \omega\right) d \mu_{1}}{\pi R^{2}\left(1+M_{O R}\right)^{2}\left(1-M_{\bar{V} R}\right)^{2}\left(1-M_{W R}\right)^{2}\left(I-M_{\bar{V}_{1}} \sin \alpha\right)}
$$


Writing

$$
\Pi_{K}^{*}\left(\mu_{3}, \omega\right)=\int_{-\infty}^{\infty} \Pi_{K}\left(\mu_{1}, \mu_{3}, \omega\right) d \mu_{1},
$$

it follows from (5.41) that

$\left\langle p_{K}\left(x_{1}, x_{3}, t\right) p_{K}\left(x_{1}, x_{3}+X_{3}, t+\tau\right)\right\rangle=\int_{-\infty}^{\infty} \Pi_{K}^{*}\left(\mu_{3}, \omega\right) e^{i\left(\mu_{3} x_{3}-\omega \tau\right)} d \mu_{3} d \omega$,

and accordingly an experimental determination of $\pi$ * may be made from surface pressure correlation measurements at a fixed distance - $x_{1}$ upstream of the edge of the plate. In order to neglect the error function term in (5.40) it is necessary that the location $x_{1}$ be closer to the edge than the smallest eddy length scale of interest; in other words, $x_{1}$ defines the largest frequency, $\quad V /\left|x_{1}\right|$, for which estimates of the far field sound are possible.

A difficulty arises when we attempt to relate the surface pressure spectrum $\Pi_{I}\left(\mu_{1}, \mu_{3}, \omega\right)$, say, to the corresponding acoustic spectrum $S_{I}(\omega)$ in the No-kutta-condition case. This is because the pressure perturbation pI given by Eq. (5.11) is singular at the edge of the plate. The pressure is finite at all points of the wake $\left(x_{1}>0, x_{2} \boxminus 0\right)$, and the spectrum $\Pi_{I}$ must therefore be defined with reference to that region alone.

Confining attention to the case in which the eddy convection velocity is essentially uniform we now obtain:

$$
S_{I}(\omega) \simeq \frac{2 M_{\bar{V}} \sin \alpha \cdot \sin ^{2}\left(\frac{\bar{\theta}}{2}\right) \cos \beta \int_{-\infty}^{\infty} \pi_{I}\left(\mu_{1}, \frac{\omega \cos \alpha}{c}, \omega\right) d \mu_{1}}{\pi R^{2}\left(1+M_{O R}\right)^{2}\left(I-M_{\overline{V R}}\right)^{2}\left(1-M_{\bar{V}_{1}} \sin \alpha\right)(1-\bar{\sigma})^{2}} .
$$

with $\bar{\sigma}=W / \bar{V}$. 
A comparison of (5.48), (5.51) indicates that at small mean flow Mach numbers a dimensionless function $F(\omega \delta * / W)$ may be introduced with the properties:

$$
\begin{aligned}
F & \rightarrow 0 \text { as } \frac{\omega \delta^{*}}{W}+\infty \\
& +\bar{\sigma} \text { as } \frac{\omega \delta^{*}}{W} \rightarrow 0,
\end{aligned}
$$

where $\omega \delta^{*} / W$ is a Strouhal number based on the laminar sublayer thickness $\delta^{*}$ near the trailing edge. This function may be taken to characterize the extent to which the Kutta condition is fulfilled in practice, so that the composite low Mach number relation between the surface spectrum $\Pi\left(\mu_{1}, \mu_{3}, \omega\right)$ and the acoustic spectrum $S(\omega)$ may be expected to assume the form

$$
S(\omega)=\frac{2 M_{\bar{V}} L \sin \alpha \sin ^{2}\left(\frac{\bar{\theta}}{2}\right) \cos \beta \int_{-\infty}^{\infty} \pi\left(\mu_{1}, \frac{\omega \cos \alpha}{c}, \omega\right) d \mu_{1}}{\pi R^{2}\left\{I-F\left(\frac{\omega \delta^{*}}{W}\right)\right\}^{2}} .
$$

The absence of information regarding the exact frequency dependence of the transition function $F(\omega \delta / W)$ is a reflection of the possible errors involved in predicting the level of the edge noise. The nature of the error is precisely that discussed at the end of Sec. 5.3 and illustrated in Table 1, and may easily amount to $10 \mathrm{~dB}$ or more.

SECTION 5.5 The Evanescent Wave Theory of Chandiramani and Chase

The analyses of Chandiramani (1974) and Chase (1975) discussed in Sec. 3 are such that the perturbation pressure remains finite at the edge of the plate. It is natural, therefore, to identify the Kutta condition result $S_{K}(\omega)$ of (5.46) with the generalization of their theory to situations in which the mean flow wets the surface of the plate, because when this occurs the pressure is finite only when the full Kutta condition is imposed. 
In order to exhibit the effect of forward flight recall that the far field spectrum must be expressed in terms of the emission time coordinates $(r, \theta, \Phi)$ of $(5.35)$. Note that the frequency $\Omega$ observed relative to the ground is related to $\omega$, that measured by an observer fixed with respect to the plate, by

$$
\Omega=\frac{\omega}{\left(1+M_{O R}\right)}
$$

When attention is confined to the approximate formulae (5.48), (5.51) valid when there exists an essentially uniform eddy convection velocity $\underset{\sim}{V}(z) \cong \bar{v}$, we obtain in the flyover plane $(\Phi=0)$ :

(i) Kutta condition applied:

$$
\begin{aligned}
S_{K r}(\Omega) & =\frac{2 M_{\bar{v}} L\left(I-M_{0}+M_{\nabla_{1}}\right) \sin ^{2}\left(\frac{\theta}{2}\right) \cos \beta}{\pi r^{2}\left[1+\left(M_{0}-M_{W_{1}}\right) \cos \theta\right]^{2}\left[1+\left(M_{0}-M_{\bar{V}_{1}}\right) \cos \theta\right]^{2}} \\
& \times \int_{-\infty}^{\infty} \pi_{K}\left[\mu_{1}, 0, \Omega\left(I+M_{0} \cos \theta\right)\right] d \mu_{1}
\end{aligned}
$$

(ii) No Kutta condition:

$$
\begin{aligned}
S_{I r}(\Omega) & =\frac{2 M_{\bar{V}} L\left(I-M_{0}+M_{\bar{v}_{1}}\right) \sin ^{2}\left(\frac{\theta}{2}\right) \cos \beta}{\pi r^{2}(1-\bar{\sigma})^{2}\left[I+M_{0} \cos \theta\right]^{2}\left[I+\left(M_{0}-M_{\bar{v}_{1}}\right) \cos \theta\right]^{2}} \\
& \times \int_{-\infty}^{\infty} \Pi_{I}\left[\mu_{1}, 0, \Omega\left(1+M_{0} \cos \theta\right)\right] d \mu_{1} .
\end{aligned}
$$

Integration of $(5.54),(5.55)$ over all frequencies $\Omega$ yields the parametric dependencies of the far field sound on the root mean 
square turbulence velocity $v$ and the flight and source convection Doppler factors obtained previously in the analogous formulae $(5.36),(5.37)$.

Chandiramani's (1974) analysis involves the approximation

$$
\int_{-\infty}^{\infty} \pi\left(\mu_{1}, \frac{\omega \cos \alpha}{c}, \omega\right) d \mu_{1} \simeq \int_{-\infty}^{\infty} \pi\left(\mu_{1}, 0, \omega\right) d \mu_{1}=\ell_{3} \phi_{f}\left(\frac{\omega \delta}{\bar{V}}\right), \quad(5.56)
$$

where $\ell_{3}$ is a transverse eddy length scale and $\phi_{f}(\omega \delta / \bar{V})$ is the frequency spectrum of the surface pressure fluctuations close to the edge of the plate. Substitution of (5.56) into (5.54) indicates that the acoustic spectrum $S_{\mathrm{Kr}}(\Omega)$ has the same shape as the surface pressure spectrum $\phi_{f}$, provided $l_{3}$ does not vary significantly with $\omega$. This is in accord with an interesting set of experimental results obtained by Yu and Tam (1977) using a high aspect ratio wall jet. Observations of this quasi-two-dimensional flow revealed the presence of large scale vortical structures centered in the upper turbulent shear layer, and translating at approximately $0.6 \mathrm{U}_{\mathrm{J}}, \mathrm{U}_{\mathrm{J}}$ being the nozzle exit velocity. The interaction of these vortices with the trailing edge induced vortex shedding, in a manner consistent with the satisfaction of the Kutta condition, accompanied by the production of a fairly coherent sound field whose directional characteristics resembled that predicted for a half-plane. Figure 6 illustrates the strong similarity between the observed surface pressure frequency spectrum close to the trailing edge and that in the far field at $\theta=90^{\circ}$.

The formulation proposed by Chase (1975) corresponds to adopting an assumed functional form for the wavenumber spectrum $f\left(\mu_{1}, \mu_{3}, z\right)$ of the aerodynamic sources, viz.

$$
f\left(\mu_{1}, \mu_{3}, z\right)=\frac{\bar{c}_{U} \rho_{0}^{2} v^{2} \bar{V}^{2} \delta^{4}|\underset{\sim}{n} \cdot \underset{\sim}{\mu}|^{3}}{\left\{1+\left(\mu_{1}^{2}+\mu_{3}^{2}\right) \delta^{2}+\frac{(z-h)^{2}}{\left.(\underset{\sim}{\sim} / \underset{\sim}{\sim} \cdot)^{\bar{V}}\right)^{2}}\right\}^{U+1 / 2}}
$$

In this expression $\bar{c}_{\mu}$ is a numerical coefficient and $2 v / \mu \cdot \overrightarrow{\mathrm{V}}$ is the effective inner length scale, perpendicular to the plañe of the plate, of an eddy of wavenumber $\underset{\sim}{\mu}$. It is assumed that $v / \bar{v}<<1$, 

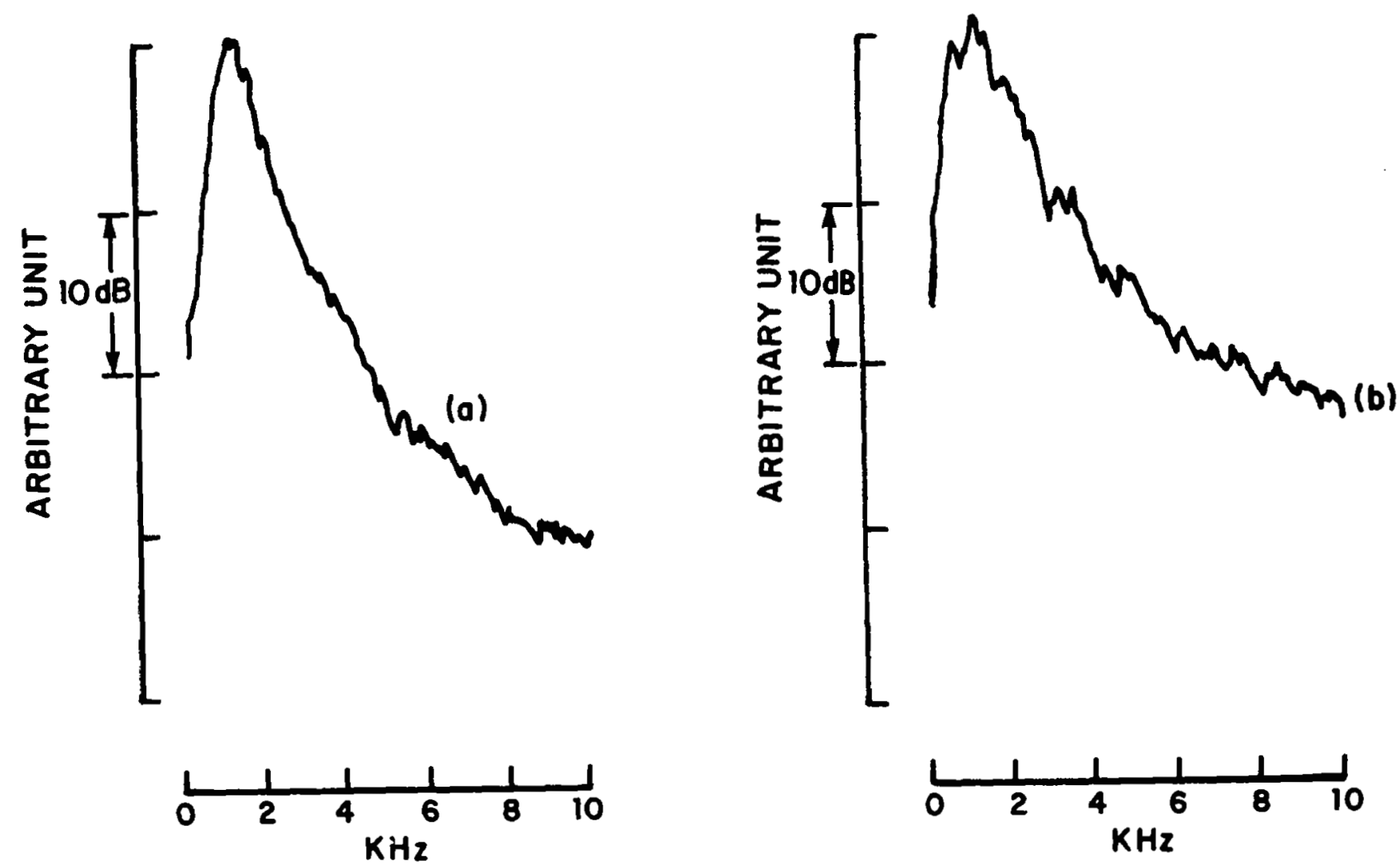

FIG. 6. COMPARISON OF THE SURFACE PRESSURE SPECTRUM NEAR THE TRAILING EDGE (a) AND THE FAR FIELD ACOUSTIC SPECTRUM (b) AT $\theta=90^{\circ}$ FOR THE EDGE NOISE EXPERIMENT OF YU AND TAM (1977). 
so that $f$ is sharply peaked at the level $z=h$ in the boundary layer. Substitution of $(5.57)$ into (5.46) yields the result (3.5) postulated by Chase for $\Pi_{i}\left(\mu_{1}, \mu_{3}, \omega\right)$, and use of this in the Kutta condition spectrum formula (5.48) gives the following generalization of Chase's (1975) theory to include effects of source motion and forward flight:

$$
S_{K r}(\Omega)=\frac{S\left[\Omega\left(I+M_{0} \cos \theta\right), r, \theta\right]\left(I-M_{0}+M_{\bar{V}_{1}}\right)}{\left[I+\left(M_{0}-M_{W_{1}}\right) \cos \theta\right]^{2}\left[I+\left(M_{0}-M_{V_{1}}\right) \cos \theta\right]^{2}} ;
$$

where $S(\omega, R, \bar{\theta})$ is the negligible Mach number result defined by the right hand side of $(3.8)$ at $\alpha=\pi / 2$ (flyover plane). 
1. The edge noise predictions of the various theories discussed in this report are essentially identical when expressed in terms of a common system of flow parameters. The extended theory of Sec. 5 indicates that at right angles to the flight path the edge noise scales as $L \ell_{3} V^{5}\left(1-M_{0}-M_{v 1}\right)$, where $L$ is the wetted span, $l_{3}$ the correlation scale parallel to the edge, f the characteristic eddy convection velocity, $M_{0}$ the flight Mach number, and $M_{v} 1$ the component of the boundary layer/wall jet Mach number perpendicular to the edge. When the effects of finite Mach number are discounted, this result is predicted by the theories of: Ffowcs Williams \& Hall (1970); Crighton (1972a); Chandiramani (1974); Chase (1972, 1975); Hayden et al. (1976); Howe $(1977)$.

2. The question of whether or not the Kutta condition should be applied in the theoretical modeling of the edge noise mechanism remains unresolved. The SPL predicted by the No-Kutta condition formulation exceeds that predicted when the Kutta condition is imposed by a factor (1 $W / V)^{-2}$, where $W$ is the near wake velocity and $V$ the characteristic eddy convection velocity in the boundary layer/wall jet, a difference which can amount to $10 \mathrm{~dB}$ or more. This critical dependence on the edge condition points to an urgent need for an experimental clarification of the precise conditions prevailing at the edge over the relevant ranges of Mach number and frequency. Chase's (1975) analysis and the experiments of Yu \& Tam (1977) suggest that the appropriate condition is weighted marginally towards the Kutta condition case.

3. Forward flight and aerodynamic source motion are characterized by a dependence of the SPL on five Doppler factors. In the No-Kutta-condition case three are associated with the flight Mach number $M_{0}$, and two with the motion of the boundary layer/wall jet sources. In the Kutta condition case, there is one Doppler factor due to flight, and two each for the motion of the incident and shed vorticity sources.

4. A simple practical relation exists between the far field acoustic spectrum and the measurable hydrodynamic surface pressure spectrum close to the trailing edge - the latter obtained from correlation measurements with respect to time and to spatial separations parallel to the edge of the plate - only when the eddy convection velocity of the incident boundary layer/wall jet turbulence is 
effectively constant, although that velocity need not equal the near wake convection velocity of the shed vorticity. In most cases it seems likely that there will be a predominant eddy convection velocity, and an experimental determination of the wavenumber-frequency spectrum $\Pi_{K}^{*}\left(\mu_{3}, \omega\right)$ of Eqs. $(5.49,5.50)$ would then permit the acoustic spectrum to be estimated via Eq. (5.48). Existing experimental data (c.f. Hayden et al. 1976) are not ideal for comparison with theory since they involve surfaces which are a poor approximation to the semi-infinite geometry and for which the flow fields have generally been inadequately specified, and correlations between interrelated parameters have not always been made.

5. The theory described in Sec: 5 is expected to be valid even at high subsonic Mach numbers provided that it is permissible to neglect compressible effects in the specification of the aeroacoustic sources. This Mach number range is of considerable practical importance, and it would now seem to be appropriate to attempt to examine the additional effect of compressibility both theoretically and by means of a sequence of definitive experiments. 


\section{REFERENCES}

Amiet, R.K. (1976), "Noise due to Turbulent Flow past a Trailing Edge," J. Sound \& Vib. $47,387-393$.

Bowman, J.J., Senior, T.B.A. \& Uslenghi, P.L.E. (1969), Electromagnetic and Acoustic Scattering by Simple Shapes, North Holland.

Chandiramani, K.L. (1974), "Diffraction of evanescent waves with Applications to Aerodynamically Scattered Sound and Radiation from Unbaffled Plates," J. Acoust. Soc. Am. 55, $19-29$.

Chase, D.M. (1972), "Sound radiated by Turbulent Flow off a Rigid Half-Plane as obtained from a Wavevector spectrum of Hydrodynamic Pressure," J. Acoust. Soc. Am. 52, 1011-1023.

Chase, D.M. (1975), "Noise radiated from an Edge in Turbulent Flow," AIAA J. 13, 1041-1047.

Corcos, G.M. (1964), "The Structure of the Turbulent Pressure Field in Boundary-Layer Flows," J. Fluid Mech. 18, 353-378.

Crighton, D.G. (1972a), "Radiation from Vortex Filament Motion near a Half-Plane," J. Fluid Mech. 51, 357-362.

Crighton, D.G. (1972b), "Radiation Properties of a Semi-Infinite Vortex Sheet," Proc. Roy. Soc. Lond. A 330, 185-198.

Crighton, D.G. (1975), "Scattering and Diffraction of Sound by Moving Bodies," J. Fluid Mech. 72, 209-227.

Crighton, D.G. \& Leppington, F.G. (1970), "Scattering of Aerodynamic Noise by a Semi-Infinite Compliant Plate," J. Fluid Mech. 43, 721-736.

Crighton, D.G. \& Leppington, F.G. (1974), "Radiation Properties of the Semi-Infinite Vortex Sheet: the Initial Value Problem," J. Fluid Mech. 64, 393-414.

Curle, N. (1955), "The Influence of Solid Boundaries upon Aerodynamic Sound," Proc. Roy. Soc. Lond. A231, 505-514.

Davis, S.A. (1975), "Theory of Discrete Vortex Noise," AIAA J. $13,375-380$.

Fethney, P. (1975), "An Experimental Study of Airframe Self-Noise," AIAA paper No. 75-511. 
Ffowcs Williams, J.E. \& Hall, L.H. (1970), "Aerodynamic Sound Generation by Turbulent Flow in the Vicinity of a Scattering Half-Plane," J. Fluid Mech. 40, 657-670.

Fink, M.R. (1975), "Experimental Evaluation of Trailing Edge and Incidence Fluctuation Noise Theories," AIAA paper No..75-206.

Fisher, M.J. \& Morfey, C.L. (1976) AGARD-LSP-80 on Aerodynamic Noise, Paper No. 3, Jet Noise.

Grosche, F.R. (1970), "On the Generation of Sound Resulting from the Passage of a Turbulent Air Jet over a Flat Plate of Finite Dimensions," Roy. Aircraft Est. Lib. Trans., No. 1460 .

Hardin, J.C. (1976), "Airframe Self-Noise - Four Years of Research," NASA Tech. Memo, NASA TM X-73908.

Hayden, R.E. (1969), "Sound Generation by Turbulent Wall Jet Flow over a Trailing Edge," M.S. Thesis, Purdue University.

Hayden, R.E., Fox, H.L. \& Chanaud, R.C. (1976), "Some factors influencing Radiation of Sound from Flow Interaction with Edges of Finite Surfaces," NASA CR-145073.

Howe, M.S. (1975), "Contributions to the Theory of Aerodynamic Sound, with Application to Excess Jet Noise and the Theory of the Flute," J. Fluid Mech. 71, 625-673.

Howe, M.S. (1976), "The Influence of Vortex Shedding on the Generation of Sound by Convected Turbulence," J. Fluid Mech. $76,711-740$.

Howe, M.S. (1977), "The Effect of Forward Flight on the Diffraction Radiation of a High Speed Jet," J. Sound \& Vib. $50,183-193$.

Jones, D.S. (1972), "Aerodynamic Sound due to a Source near a Half-Plane," J. Inst. Math. Applic. 9, 114-122.

Levine, H. (1975), "Acoustical Diffraction Radiation," Philips Res. Repts. 30, 240-276.

Lighthill, M.J. (1952), "On Sound Generated Aerodynamically. I. General Theory," Proc. Roy. Soc. Lond. A 211, 564-587.

Morgan, J.D. (1974), "The Interaction of Sound with a Semi-Infinite Vortex Sheet," Q. Jl. Mech. Appl. Math. 27, 465-487. 
Noble, B. (1958), Methods based on the Wiener-Hopf Technique, Oxford: Pergamon.

Orszag, S.A. \& Crow, S.C. (1970), "Instability of a Vortex Sheet Leaving a Semi-Infinite Plate," Stud. Appl. Math. 49, $167-181$.

Powell, A. (1959), "On the Aerodynamic Noise of a. Rigid Flat Plate Moving at Zero Incidence," J. Acoust. Soc. Am. 31, 1649-1653.

Scharton, T., Pinkel, B. \& Wilby, J. (1973), "A Study of Trailing Edge Blowing as a Means of Reducing Noise Generated by the Interaction of Flow with a Surface," NASA CR-132270.

Tam, C.K.W. \& Reddy, N.N. (1977), "Sound Generated in the Vicinity of the Trailing Edge of an Upper Surface Blown Flap," J. Sound \& Vib. 52, 211-232.

Tam, C.K.W. \& Yu, J.C. (1975), "Trailing Edge Noise," AIAA paper No. $75-489$.

Watson, G.N. (1966), A Treatise on the Theory of Bessel Functions, Cambridge Univ. Préss."

Yu, J.C. \& Tam, C.K.W. (1977), "An Experimental Investigation of the Trailing Edge Noise Mechanism," AIAA paper №. 77-1291. 


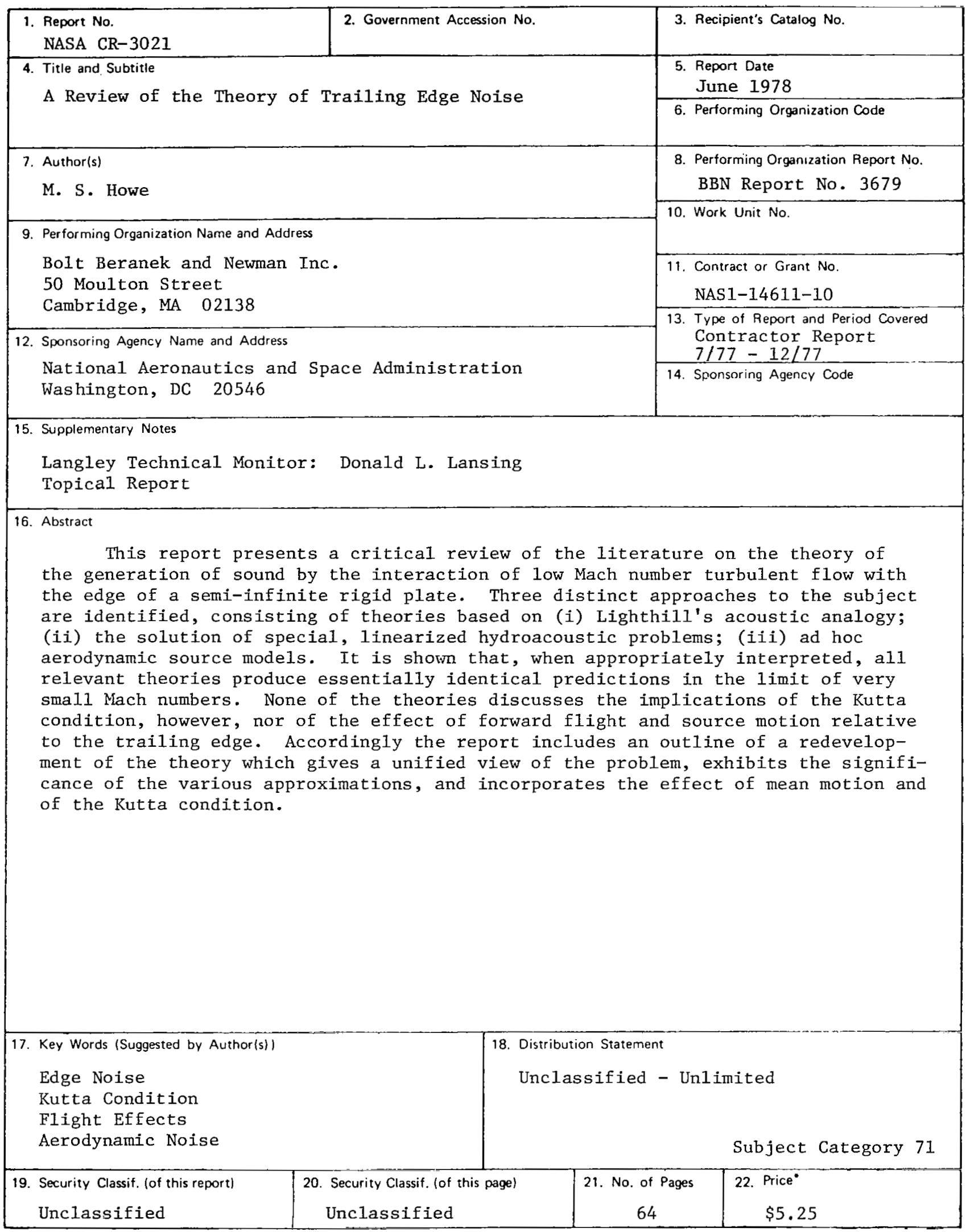

* For sale by the National Technical Information Service, Sprıngfield, Virgını 22161 